\title{
Spatial Pattern Analysis of Strawberry Leaf Blight in Perennial Production Systems
}

\author{
W. W. Turechek and L. V. Madden
}

Department of Plant Pathology, The Ohio State University, Ohio Agricultural Research Development Center, Wooster 44691. Accepted for publication 25 January 1999.

\begin{abstract}
Turechek, W. W., and Madden, L. V. 1999. Spatial pattern analysis of strawberry leaf blight in perennial production systems. Phytopathology 89:421-433.

Spatial pattern of the incidence of strawberry leaf blight, caused by Phomopsis obscurans, was quantified in commercial strawberry fields in Ohio using statistics for heterogeneity and spatial correlation. For each strawberry planting, two transects were randomly chosen and the proportion of leaflets (out of 15) and leaves (out of five) with leaf blight symptoms was determined from $N=49$ to 106 (typically 75) evenly spaced sampling units, thus establishing a natural spatial hierarchy to compare patterns of disease. The beta-binomial distribution fitted the data better than the binomial in 92 and $26 \%$ of the 121 data sets over 2 years at the leaflet and leaf levels, respectively, based on a likelihood ratio test. Heterogeneity in individual data sets was measured with the index of dispersion (variance ratio), $\mathrm{C}(\alpha)$ test, a standard normal-based test statistic, and estimated $\theta$ parameter of the beta-binomial. Using these indices, overdispersion was detected in approximately 94 and $36 \%$ of the data sets at the leaflet and leaf levels, respectively. Estimates of the slope from the bi-

leaflet and leaf levels for both years, indicating that degree of heterogeneity was a function of incidence. A covariance analysis indicated that cultivar, time, and commercial farm location of sampling had little influence on the degree of heterogeneity. The measures of heterogeneity indicated that there was a positive correlation of disease status of leaflets (or leaves) within sampling units. Measures of spatial association in disease incidence among sampling units were determined based on autocorrelation coefficients, runs analysis, and a new class of tests known as spatial analysis by distance indices (SADIE). In general, from 9 to $22 \%$ of the data sets had a significant nonrandom spatial arrangement of disease incidence among sampling units, depending on which test was used. When significant associations existed, the magnitude of the association was small but was about the same for leaflets and leaves. Comparing test results, SADIE analysis was found to be a viable alternative to spatial autocorrelation analysis and has the advantage of being an extension of heterogeneity analysis rather than a separate approach. Collectively, results showed that incidence of Phomopsis leaf blight was primarily characterized by small, loosely aggregated clusters of diseased leaflets, typically confined within the borders of the sampling units.
\end{abstract} nary power law were significantly $(P<0.01)$ greater than 1 and estimates of the intercept were significantly greater than $0(P<0.01)$ at both the

Strawberry leaf blight is a foliar disease that can cause significant damage to the strawberry plant (Fragaria $\times$ ananassa Duchesne) (25). The extent of direct losses attributed to this disease is not known. However, the disease is responsible for weakening and, thus, predisposing the plant to infection by pathogens that cause severe disease (7). The causal agent of leaf blight is the fungus Phomopsis obscurans (Ellis \& Everh.) Sutton. P. obscurans overwinters on infected plant parts and in soil debris. The pathogen primarily attacks older leaves, and typical disease development begins in late spring or early summer in northern U.S. states. In some years, high disease intensity may develop in which the stolons, petioles, and fruit become infected. Pycnidia form in the interior of older lesions and produce masses of conidia, which are typically dispersed by rain splash.

Leaf blight is controlled through cultural practices that reduce moisture on the plant (e.g., choosing planting sites with good soil drainage and full exposure to the sun), managing runner growth, weed control, and fungicides $(6,8)$. In the northern growing regions of the United States, where strawberries are grown as a perennial crop, renovation (mowing down the leaves after harvest season) is a common practice that may control foliar pathogens. There are no reported Phomopsis-resistant cultivars (7).

Little is known about the spatial pattern (dispersion) of diseased leaflets and leaves in commercial fields. Such information is needed

Corresponding author: L. V. Madden; E-mail address: Madden.1@ osu.edu

Publication no. P-1999-0301-02R

(C) 1999 The American Phytopathological Society
Additional keywords: quantitative epidemiology, statistical ecology. to fully understand disease dynamics $(9,26)$, develop more accurate sampling plans (30), better assess crop loss in relation to disease intensity (16), and design and analyze experiments more efficiently (20). There is a variety of statistical tools available to quantify spatial pattern $(3,5,41)$ that can be categorized based on the general type of analysis (point pattern versus correlation) (50) or on whether the data consist of mapped or unmapped observations (5). Pointpattern analysis is conducted using either the distances between individuals, for cases in which individuals are intensely mapped $(5,9,26)$, or the counts of individuals within sampling units such as quadrats $(26,41,50)$. All of the general methods based on counts per sampling unit provide direct measurements of heterogeneity or variability of the data among sampling units, which is a function of the spatial association of the individuals within sampling units (28), but they do not explicitly define the spatial arrangement of the sampling-unit counts, unless several sampling units are merged in a series of steps $(1,50)$.

As an alternative to the point-pattern methods, various forms of spatial statistics such as spatial autocorrelations and semivariograms $(4,12,52)$ can be used for obtaining explicit information on the degree of association of disease intensity among sampling units (26). In particular, these correlation-based methods yield different results for different arrangements of counts within a field, although they are not specifically developed for counts of organisms or diseased individuals within sampling units. A new method was recently developed called spatial analysis by distance indices (SADIE) $(36,38)$. SADIE is a class of tests developed to quantify spatial pattern in the spirit of spatial autocorrelation, but it uses data in the form of point patterns $(36,39)$. SADIE, like spatial autocorrelation analy- 
sis, requires that the relative position of the sampling units (but not necessarily the individuals) be known so that they can be mapped onto a Cartesian plane. Point-pattern and correlation-type results can jointly be used to more fully interpret patterns and possibly describe the biological phenomenon responsible for generating the observed pattern $(2,26)$.

The purpose of this research was to (i) quantify the spatial pattern (heterogeneity and spatial arrangement) of the incidence of strawberry leaf blight in perennial production systems; (ii) evaluate SADIE as a new class of tests for the analysis of spatial pattern of leaf blight; and (iii) determine if spatial pattern and heterogeneity were associated with crop conditions such as cultivar and assessment time. It is important to emphasize four defining aspects of this research. First, the spatial data were collected from commercial fields with no experimental manipulation, except for limited plant handling to assess disease. This study would be classified as a 'mensurative' experiment by Hurlbert (24). Second, data were collected with sparse sampling (23) of individual leaflets or leaves but with known position of the sampling units within fields. Mapping of individual leaflets would be extremely difficult except for a very small area and limited sampling times. Third, the statistical methods used were based on the recent developments in analysis of distributions and in heterogeneity of disease incidence $(21,28$, 46). Similarities of the test results for the conditions of this study were determined and related to the properties of the calculated statistics. Fourth, a relatively large number of data sets were obtained over 2 years so that we could assess the effects of time of year, cultivar, and farm location on spatial heterogeneity and pattern of disease incidence.

\section{MATERIALS AND METHODS}

Data collection. Naturally occurring epidemics of leaf blight of strawberry were monitored over 2 years, 1996 and 1997, at two different commercial strawberry farms in Ohio. Farm 1 was located near Wooster, $\mathrm{OH}\left(40^{\circ} 49^{\prime} 06^{\prime \prime} \mathrm{N}, 81^{\circ} 55^{\prime} 58^{\prime \prime} \mathrm{W}\right)$, and farm 2 was located approximately $151 \mathrm{~km}$ southwest of Wooster in Darbydale, $\mathrm{OH}\left(39^{\circ} 51^{\prime} 13^{\prime \prime} \mathrm{N}, 83^{\circ} 10^{\prime} 59^{\prime \prime} \mathrm{W}\right)$. Each farm had several perennial plantings (fields) comprised of 10 to 20 rows of strawberry. The perennial plantings ranged in age from 1 to 5 years. Plants were grown in a matted row system approximately $0.3 \mathrm{~m}$ wide grown on 1 -m centers. 'Earliglow' was the dominant cultivar grown at both locations. Other cultivars rated for disease included 'Honeoye', 'Jewel', 'Allstar', 'Delmarvel', and 'Primetime'. All cultivars are day-length sensitive (June bearers) and are susceptible to P. obscurans.

For each field and assessment time, a single row was randomly chosen as the primary transect and a short secondary transect was arbitrarily chosen to approximately bisect the field. Sampling units consisted of five leaves of three leaflets each that were rated for the presence or absence of leaf blight symptoms. The initial sampling unit was arbitrarily chosen within the first 20 "steps" of the primary transect and each subsequent sampling unit was located at a distance of three steps $(\sim 2.5 \mathrm{~m})$ from the previous sampling unit, assuring approximately evenly spaced sampling units. A sampling unit in each row also was sampled along the secondary (bisecting) transect. Five leaves were chosen in each sampling unit (without regard to disease status) adjacent to where the rater stopped, and each of the three leaflets per leaf were rated for the presence or absence of leaf blight symptoms. This is a form of cluster sampling in which the "clusters" (sampling units) were chosen systematically and all individuals (e.g., leaflets) per sampling unit (n) were assessed (21). Disease incidence was thus assessed at three levels in a spatial hierarchy: the leaflet level, $n=n_{\mathrm{t}}=15$ (15 leaflets per sampling unit); the leaf level, $n=n_{\mathrm{f}}=5$; and the level of the sampling unit, $n=n_{\mathrm{su}}=1$. If there was a Phomopsis lesion on any one of the leaflets in a leaf, then the leaf was rated as diseased. At the sampling-unit level, the cluster sampling reduces to a simple systematic sample. Most presented analyses are based on the leaflet level. The number of sampling units, $N$, varied from 49 to 106, depending upon the field size, but was typically 75 .

In 1996, sampling started on 20 June and continued until 13 September. These dates encompassed time of fruit development, harvesting, renovation, and regrowth of vegetation. Not all fields were sampled the same number of times. The same leaves were not necessarily sampled in different assessments in the same field. In 1997, sampling started on 29 April and continued until 26 September.

In 1997, two additional systematic cluster sampling plans were used to determine if a significant amount of spatial information was going undetected in the standard three-step transect. Data from rows more intensely sampled than the standard samples were collected. An intensively sampled transect differed from a standard transect, defined above, in that the distance between neighboring sampling units was one step $(\sim 0.85 \mathrm{~m})$. In these transects, $N$ varied from 200 to 250 over approximately the same distance as for standard transects. Additionally, two-dimensional (2D) sampling was done in which 10 sampling units were chosen in each of 10 adjacent rows per field. There were three steps between sampling units within rows. In this sampling plan, $N=100$.

Heterogeneity analyses. Distribution and indices analyses. The beta-binomial $(18,28)$ and the binomial distributions were fitted to the data using the computer program BBD (27) for each individual field assessment. The beta-binomial has two parameters, $p$, which is the expected probability of disease, and $\theta$, a measure of the variation in disease incidence per sampling unit. The binomial has a single parameter representing the probability of disease $(\pi)$. A good fit to the binomial distribution is suggestive of a random spatial pattern of disease incidence, while a good fit to the beta-binomial is suggestive of an aggregated spatial pattern of disease incidence. Standard $\chi^{2}$ goodness-of-fit tests (18) were calculated for each distribution after disease incidence classes (e.g., 0 to 15 diseased leaflets) were pooled so that all expected frequencies exceeded five. The degrees of freedom (df) are given by (number of disease classes after pooling) (number of parameters) - 1. Maximum likelihood as well as moment estimates of the beta-binomial parameters were calculated, although the maximum likelihood estimates were used in all calculations when the maximum likelihood method was successful. $t$ tests were used to determine if maximum likelihood estimates of $\theta$ differed significantly from 0. A log-likelihood ratio test statistic (LRS) was calculated for each data set to determine if the beta-binomial fitted the data better than the binomial (51). LRS $=2 \cdot[\ell(\mathbf{x} ; \hat{p}, \hat{\theta})-\ell(\mathbf{x} ; \hat{\pi})]$, in which $\ell(\mathbf{x} ; \hat{p}, \hat{\theta})$ and $\ell(\mathbf{x} ; \hat{\pi})$ are the log-likelihoods for the beta-binomial and binomial, respectively, $\mathbf{x}$ is a vector of the observed number of diseased individuals per sampling unit, and $\pi, p$, and $\theta$ are as previously defined. LRS has a $\chi^{2}$ distribution with $1 \mathrm{df}$ under the null hypothesis of no difference between the two log-likelihoods (i.e., the beta-binomial does not characterize the frequency distribution of diseased leaflets or leaves any better than the binomial) (51).

For each field and assessment date, the index of dispersion, $D$, was calculated as the ratio of the observed variance of $x$ among the sampling units to the expected binomial variance (28). For a given level of incidence, the effect of disease aggregation on the observed variance is to inflate it above the expected binomial variance. Therefore, values of $D$ greater than 1 suggest spatial aggregation (at some scale). $(N-1) \cdot D$ has a $\chi^{2}$ distribution under the null hypothesis of randomness $(N-1 \mathrm{df})$. Sun and Madden (46) recently showed that $D$ also has an asymptotic normal distribution under the null hypothesis of randomness with a mean of 1 and variance equal to 2(1$1 / n) /(N-1)$. Consequently, a standard normal test statistic $\left(z_{\mathrm{sm}}\right)$ can be calculated from $D, N$, and $n$, and this value can be used as an alternative way to test for randomness. Moreover, a so-called $\mathrm{C}(\alpha)$ test was used to test for overdispersion (48). The test statistic has a standard normal distribution under the null hypothesis and is given by $z_{\mathrm{C}(\alpha)}=[(n N-1) D-n N] /\left[2 N\left(n^{2}-n\right)\right]^{1 / 2}$. However, the alternative hypothesis is not just overdispersion but overdispersion described by the beta-binomial. This is a one-sided test, thus, the null hypothesis is rejected when $z_{\mathrm{C}(\alpha)}>1.645$. 
Power law analysis. In 1996 and 1997, $67\left(N_{\mathrm{T}}=67\right)$ and $54\left(N_{\mathrm{T}}=\right.$ 54 ) independent disease assessments were made, respectively. The binary form of Taylor's power law $(19,49)$ was used as an assessment of heterogeneity or overdispersion across all data sets. Separate analyses were conducted in 1997 for the intensive and 2D sampling plans. Unlike the distributional approaches described above, which are used to assess the degree of heterogeneity found in single data sets, the power law collectively utilizes the variability found in all the data sets considered in order to characterize heterogeneity and show if degree of heterogeneity is related to disease incidence. The power law, as rewritten by Hughes and Madden (17), can be simply expressed as a relationship between two variances, the observed sample variance of diseased leaflets $\left(v_{\mathrm{obs}}\right.$, second moment about the mean) and the theoretical variance of a random distribution $\left(v_{\text {ran }}\right)$, and can be written as

$$
v_{\text {obs }}=A_{x} v_{\text {ran }}{ }^{b}
$$

in which $A_{x}$ and $b$ are parameters. With binary data, $v_{\text {ran }}$ is the binomial variance, $n p(1-p)$. By taking logarithms of both sides of equation of 1 , we obtain

$$
\ln \left(v_{\text {obs }}\right)=\ln \left(A_{x}\right)+b \ln [n \hat{p}(1-\hat{p})]
$$

in which $\ln \left(A_{x}\right)$ and $b$ are the intercept and slope of a straight line, respectively. In fitting equation 2 to the data by regression analysis, we used the estimated $p$ from the beta-binomial distribution $(\hat{p})$ and the moment estimate of $v_{\text {obs }}$ for each data set. When $A_{\mathrm{x}}$ and $b$ are both equal to 1 , randomness as described by the binomial distribution is indicated. When $b=1$ and $A_{x}>1$, there is overdispersion, but the degree of overdispersion for each data set (e.g., $\theta$ ) does not depend on $p$. When $b$ and $A_{x}$ are greater than 1 , the degree of overdispersion or heterogeneity changes with $p$.

A covariance analysis was conducted to determine the effect of the factors (class variables) year, cultivar, farm location, and assessment date on the slope $(b)$, intercept $\left(\ln \left[A_{x}\right]\right)$, or both, of the power law (equation 2). Three cultivar categories were considered: 'Earliglow', 'Honeoye', and 'others' (all other cultivars). There were not enough data sets for 'Allstar', 'Jewel', 'Delmarvel', and 'Primetime' to realistically test for their individual effects on the regression parameters. For the date factor, three categories were created to approximately represent the time periods: 1 , from the beginning of sampling to crop renovation; 2, the 2-week period following renovation (a time when foliage may be more resistant to infection); and 3, from 2 weeks after renovation to the end of the growing season. Since husbandry practices varied considerably between farms and among fields within farms, these date categories are only approximations. Generalized Linear Interactive Modeling (GLIM) (version 3.77; Numerical Algorithms Group, Ltd., Oxford, London) was used for the regression analysis. The continuous variable $\ln [n \hat{p}(1-\hat{p})]$ was always included in the model first, and then the factors were added. The covariance analysis was done for each year separately; a separate covariance analysis was then done to determine if the year factor affected $\ln \left(A_{x}\right)$ or $b$. Because the power law is based on the principle that heterogeneity $\left(v_{\mathrm{obs}}\right)$ is related to disease incidence, the test of a factor in the covariance analysis is not an indication of whether or not the factor had an influence on disease incidence or heterogeneity, but on whether or not the factor affected the degree of heterogeneity after first correcting for the relationship between heterogeneity and $p$.

The beta-binomial parameter $\theta$ can be written in terms of the power law estimates $(17,28)$. The relationship can be expressed as

$$
\theta=[a-f(p) / n] /[f(p)-a]
$$

in which $f(p)=[p(1-p)]^{1-b}$ and $a=A_{x} n^{b-2}$ (28). The expression for $a$ is based on the binary power law for counts (in which each $x$ is between 0 and $n$ ) and is different from the expression for $a$ in Madden and Hughes (28), which is for the power law results based on proportions ( $a=A_{p} n^{-b}$, with $A_{p}$ being a more specific symbol for their proportion-based results). Equation 3 represents a curve with maximum value at $p=0.5$ when $b>1$, and a horizontal line when $b=1$ (28). This equation provides a link between the distributional approach and the empirical relationship of the power law that is unique for binary data. This is similar to the link between the Adès distribution and the power law for unbounded count (e.g., animal counts) data (40).

Correlation-type analyses. Spatial autocorrelation. For each field and assessment date, the number of diseased leaflets (or leaves) in each sampling unit along the primary transect was transformed using the logit transformation, $\ln [y /(1-y)]$, in which $y=(x+$ $0.5) /(n+1)$ and $x$ is the number of diseased leaflets in each sampling unit containing $n$ leaflets. This corrected value of disease proportion, known as the Haldane correction $(43,44)$, was used to avoid taking the logarithm of 0 values or dividing by 0 . Spatial autocorrelation coefficients were calculated for the standard transect analysis of 1996 and 1997 and the intensive transects of 1997 with the statistical program Minitab (version 10; Minitab Inc., State College, PA). The secondary transects (the short transects of sampling units across the fields) were not used in the spatial autocorrelation analysis. For the 2D data sets, autocorrelation coefficients were calculated using STAUTO, a specialized Pascal routine developed by Reynolds and Madden (43) for 2D spatial and spatiotemporal analyses. The 'row' (comparisons within strawberry rows), 'column' (comparison across strawberry rows), and 'rook' (comparison across and within strawberry rows) proximity patterns were evaluated and binary distance weighting was used (43).

Autocorrelation analysis of the intensive transect allowed us to determine if the standard interval between sampling units (three steps) was too large for detecting association of disease incidence between proximal locations. In other words, we could use the results to determine if sampling-unit spacing affected magnitude of correlation coefficients. The data of the intensive transects were handled in the same manner as the standard transects. Besides the analysis based on all $N$ sampling units, the analysis was done for every third sampling unit starting at positions 1,2 , and 3 . This is equivalent to the analysis of the standard transects.

Runs analysis. For the data of the standard and intensive transects, ordinary and median runs analysis were performed (10). A run is a sequence of sampling units with the same disease status. For example, if 1 indicates disease and 0 indicates no disease, the sequence 1-1-0-1-0-0 contains four runs (i.e., 1-1, 0, 1, and 0-0). For median runs analysis, the median level of disease incidence in each transect was calculated. Sampling units that contained values greater than the median were coded as 1 , all others were coded as 0 . Ordinary runs analysis simply was based on sampling units with either no disease (0) or greater than zero diseased leaflets (1). No information other than presence or absence of disease in that sampling unit was needed to conduct this latter test. Runs and spatial autocorrelations are both special cases of cross-product statistics (26).

SADIE. SADIE uses the locations of sampling units and the counts of diseased individuals per unit to evaluate the spatial arrangement of diseased individuals. Like the correlation-type analyses, SADIE results reflect the spatial arrangement of disease at the level of the sampling unit and above. The difference is that, with SADIE, the analysis is conditioned on the preexisting level of heterogeneity in the data set (i.e., heterogeneity is incorporated into the null hypothesis of a random arrangement of diseased counts).

The key concept behind SADIE is the notion of a "distance" to regularity $(36,38)$. This is best illustrated by example. Consider six sampling units equally spaced along a transect that can contain a maximum number of 15 diseased leaflets each. Given a particular realization, say 12-6-0-0-0-0 diseased leaflets, SADIE computes the minimum number of "moves" of the diseased leaflets so that the arrangement of these 18 individuals is as regular as possible, in this case, 3-3-3-3-3-3. From the perspective of heterogeneity analysis, complete regularity corresponds to $v_{\mathrm{obs}}=0$. Here, the distance to regularity $\left(D_{\mathrm{r}}\right)$ is equal to 39 (if each move is con- 
sidered a move of unit distance). To determine if 39 is a particularly high or low number, SADIE performs a randomization test (37). The values of the counts in each sampling unit are randomly reassigned to new sampling units and then the distance to regularity is recalculated, e.g., 0-12-0-6-0-0 could be one of many alternative randomizations ( $D_{\mathrm{r}}=21$ for this particular randomization). This can be done a number of times to derive a frequency distribution of distances or moves. From this frequency distribution, percentiles can be calculated (e.g., $2.5 \%, 5 \%$, etc.) so that a one- or two-sided test of spatial randomness can be performed at a specified probability level (e.g., $\alpha=0.05$ ). If the distance to regularity of the observed sample is sufficiently large relative to the distances to regularity of the randomized cases, then the null hypothesis of a random spatial arrangement is rejected in favor of the alternative that the counts in the sampling units show a more aggregated (clumped, clustered) arrangement than what would be expected from a random arrangement of the counts in the sampling units. Alternatively, if the distance to regularity is sufficiently small, then the null hypothesis is rejected in favor of the alternative hypothesis that the counts in the sampling units show a more regular pattern than what would be expected from a random arrangement of the counts. Thus, aggregated arrangements tend to have large distances to regularity, while more regular arrangements tend to have smaller distances to regularity. From the above example, one can determine that the realization of data is the most aggregated possible.

An index of aggregation $\left(I_{\mathrm{a}}\right)$ based on the SADIE methodology can be written as

$$
I_{\mathrm{a}}=D_{\mathrm{r}} / E_{\mathrm{a}}
$$

in which $E_{\mathrm{a}}=$ the average (expected) distance to regularity of the randomization results. A value of 1 suggests a spatially random pattern; greater than 1, a more aggregated pattern; and a value less than 1 , a more regular pattern. However, $I_{\mathrm{a}}$ is not directly used for determining significance; only the percentages of distances from the randomizations are used.

For each year, field, and assessment date, SADIE analysis was performed using the primary transect. The intensive and 2D samples of 1997 were analyzed as well. Initially, 100 randomizations were performed for each data set. From these results, $P_{\mathrm{a}}$, the proportion of randomizations with distances to regularity larger than $D_{\text {r }}$ was obtained from the output. Samples in which $P_{\text {a }}<0.10$ or $P_{\mathrm{a}}>0.90$ were subjected to a to second set of 500 randomizations in order to more accurately estimate the achieved significance $(P$ value) of the two-sided hypothesis test of nonrandomness. Signifi- cance for the two-sided test is determined from $2 \cdot \min \left(P_{\mathrm{a}}, 1-P_{\mathrm{a}}\right)$, in which $\min (\bullet, \bullet)$ is the 'minimum' function.

\section{RESULTS}

Point-pattern approaches. Distribution analysis. The program BBD successfully calculated maximum likelihood estimates of $p$ and $\theta$ for 91 and $81.5 \%$ of the data sets at the leaflet level in 1996 and 1997, respectively. In all cases in which maximum likelihood estimation was not possible, $\hat{p}$ was less than 0.05 . At the leaf level, maximum likelihood estimates were obtained in 76.1 and $64.8 \%$ of the data sets in 1996 and 1997, respectively. Of the 35 data sets in which maximum likelihood estimation was not possible, 22 data sets had a $\hat{p}$ less than 0.10 . It was always possible to calculate parameters using the moment method.

Where there was a sufficient number of disease classes for the test to be performed, the frequency distribution of diseased individuals could be described by the beta-binomial distribution in over $70 \%$ of the fields at the leaflet level and over $87 \%$ of the fields at the leaf level for the 2 years (Table 1). The binomial distribution described the data in less than $7 \%$ of the fields at the leaflet level and in about 65\% at the leaf level in 1996 and 1997 (Table 1). The LRS showed that the frequency distribution of diseased leaflets could be described better by the beta-binomial rather than the binomial distribution in over $87 \%$ of the data sets in 1996 and 1997 (Table 1). The frequency distribution of diseased leaves was described better by the beta-binomial distribution in only about a quarter of the data sets in 1996 and 1997. The LRS was calculated for all data sets, even when the maximum likelihood procedure did not converge. In the latter cases, moment estimates of $p$ and $\theta$ were used.

Figure 1 shows typical transect maps and frequency distributions for three fields that all had approximately $20 \%$ diseased leaflets ( $\hat{p} \approx 0.2$ ) but three different levels of heterogeneity, as represented by $\hat{\theta}$ of the beta-binomial. One can see the increasing variation in diseased leaflets with increasing $\hat{\theta}$ (Fig. 1A to $\mathrm{C}$ ) and the increasing skewness of the frequency distribution (Fig. 1D to F, solid bars) with increasing $\hat{\theta}$. The binomial provided a poor fit to all three examples, and the beta-binomial provided a very good fit for these, especially for the larger $\hat{\theta} \mathrm{s}$.

Disease incidence. Mean disease incidence of leaflets ranged from 0.0009 (one diseased leaflet in a field) to 0.78 in 1996 and from 0.0009 to 0.33 in 1997, as determined from the estimated expected probability of disease ( $\hat{p}$ ) (Fig. 2A and G). Overall, there was higher incidence in 1996 than in 1997, with median $\hat{p}$ across all the data sets in 1996 nearly triple the median in 1997. How-

TABLE 1. Percentage of data sets of Phomopsis leaf blight of strawberry in which the null hypothesis of randomness was rejected $(P \leq 0.05)$ or, for the goodness-offit tests, failed to reject the null hypothesis (with number of tested data sets in parentheses)

\begin{tabular}{|c|c|c|c|c|c|c|c|c|c|c|c|}
\hline \multirow[b]{2}{*}{ Data set } & \multicolumn{2}{|c|}{ Goodness of fit ${ }^{\mathrm{a}}$} & \multirow[b]{2}{*}{$\mathrm{LRS}^{\mathrm{b}}$} & \multirow[b]{2}{*}{$\theta^{\mathrm{c}}$} & \multicolumn{3}{|c|}{ Tests of $D^{\mathrm{d}}$} & \multirow[b]{2}{*}{$r_{1} \mathrm{e}^{\mathrm{e}}$} & \multicolumn{2}{|c|}{ Runs $^{f}$} & \multirow[b]{2}{*}{ SADIE $^{\mathrm{g}}$} \\
\hline & BBD & $\mathrm{BIN}$ & & & $\chi^{2}$ & $z_{\mathrm{sm}}$ & $z_{\mathrm{C}(\alpha)}$ & & Median & Ordinary & \\
\hline 1996 leaflets & 87.8 & 2.2( & $95.6(67)$ & 89.0 & 97.0 & 97.0( & 97.0 & $9.0(67)$ & $9.0(67)$ & 12. & 16.4 \\
\hline 1997 leaflets & $70.8(24)$ & $6.0(32)$ & $87.0(54)$ & 84.0 (44) & $88.9(54)$ & $88.9(54)$ & 90.7 (54) & $16.9(53)$ & $17.0(53)$ & $15.1(53)$ & $20.8(53)$ \\
\hline 1997 leaves & $88.9(9)$ & 68.5 (19) & $24.1(54)$ & $20.0(35)$ & $35.2(54)$ & $35.2(54)$ & $42.6(54)$ & $15.1(53)$ & $17.0(53)$ & $15.1(53)$ & $22.6(53)$ \\
\hline
\end{tabular}

${ }^{a} \mathrm{BBD}=$ beta-binomial distribution; BIN = binomial distribution. Numbers are the percentages of instances that the indicated distribution provided a good fit based on a $\chi^{2}$ goodness-of-fit test. A good fit to the BBD and a poor fit to the BIN are indicators of overdispersion. Number of tested data sets is substantially lower than for other tests, because goodness of fit can only be evaluated when there are, after pooling, at least three disease classes for BBD and two classes for BIN.

${ }^{b}$ The likelihood ratio test statistic (LRS) (51): two times the difference of the log-likelihoods for the beta-binomial and the binomial distributions. Significant results indicate that the beta-binomial provides a better fit than the binomial and, hence, the data exhibit overdispersion.

${ }^{\mathrm{c}}$ Maximum likelihood estimate of the beta-binomial aggregation parameter, $\theta$. Significant departures from zero, determined with a $t$ test, indicate overdispersion.

${ }^{\mathrm{d}}$ Index of dispersion, $D(28) \cdot \chi^{2}, z_{\mathrm{sm}}$, and $z_{\mathrm{C}(\alpha)}$ are the test statistics for nonrandomness (overdispersion) based on the chi-square (18) and standard normal distributions $\left(z_{\mathrm{sm}}[46] ; z_{\mathrm{C}(\alpha)}[48]\right)$.

${ }^{\mathrm{e}}$ First-order spatial autocorrelation coefficient, $r_{1}$, indicate significant spatial correlation in disease incidence in neighboring sampling units.

${ }^{\mathrm{f}}$ Median and ordinary runs analyses indicate significant associations in disease status in neighboring sampling units. Ordinary runs: each sampling unit is classified as disease free ( 0$)$ or diseased (1; one or more diseased leaflets) (10). Median runs: each sampling unit is classified as being below ( 0$)$ or above (1) the median incidence for the data set.

${ }^{g}$ SADIE (38) is based on the number of moves to regularity of the observed data $\left(D_{\mathrm{r}}\right)$ relative to the moves to regularity for randomizations of the observed data. $P_{\mathrm{a}}$, is the proportion of SADIE randomizations that are larger than $D_{\mathrm{r}}$ and was used to determine the significance $(P$ value $)$ of a two-sided hypothesis test of nonrandomness. Significance for this test was determined from $2 \cdot \min \left(P_{\mathrm{a}}, 1-P_{\mathrm{a}}\right)$, in which $\min (\bullet, \bullet)$ is the 'minimum' function. 
ever, the frequency distributions of $\hat{p}$ values were quite similar, with a large positive skewness. Mean disease incidence at the leaf level ranged from 0.0027 to 0.89 in 1996 and from 0.0027 to 0.51 in 1997, with median values across all data sets of 0.18 and 0.07 in 1996 and 1997, respectively (frequencies not shown).

Heterogeneity measures. The frequency distribution of $\hat{\theta}$ for diseased leaflets was about the same for both years. $\hat{\theta}$ ranged from 0 to 0.35 in 1996 and from 0 to 0.37 in 1997 (Fig. 2B and $\mathrm{H})$. There was also a slight positive skewness of values. Median $\hat{\theta}$ was about 0.1 for both years. Over $85 \%$ of these were significantly greater than 0 (Table 1), indicating substantial overdispersion. At the leaf level, $\hat{\theta}$ ranged from 0 to 0.34 in 1996 and from 0 to 0.46 in 1997, with a median $\hat{\theta}$ of about 0.04 (frequencies not shown). The distribution of values was characterized by a large positive skewness.
The index of dispersion, $D$, ranged from 0.96 to 4.94 over both years, and the frequency distribution of $D$ values was very similar for 1996 and 1997 at the leaflet level (Fig. 2C and I). Likewise, the standard normal test statistic for the $C(\alpha)$ test, $z_{C(\alpha)}$ (Fig. 2D and J), and the standard normal for the Sun and Madden (46) test, $z_{\mathrm{sm}}$ (data not shown), had very similar results for both years. Given that the maximum value of $D$ for the data sets is 15 (for $n=15$ ) and the maximum value of $z_{\mathrm{C}(\alpha)}$ is approximately 88 (for $n=15$ and $N=75$ ), it is clear that the level of heterogeneity, though significantly greater than for a random distribution, was not extremely large (Fig. 2C, D, I, and J). At the leaf level, $D$ ranged from 0.81 to 2.31 over both years, with a median of 1.18, and also had similar frequency distributions. The $\mathrm{C}(\alpha)$ test and the Sun and Madden test had results similar to $D$ (data not shown).
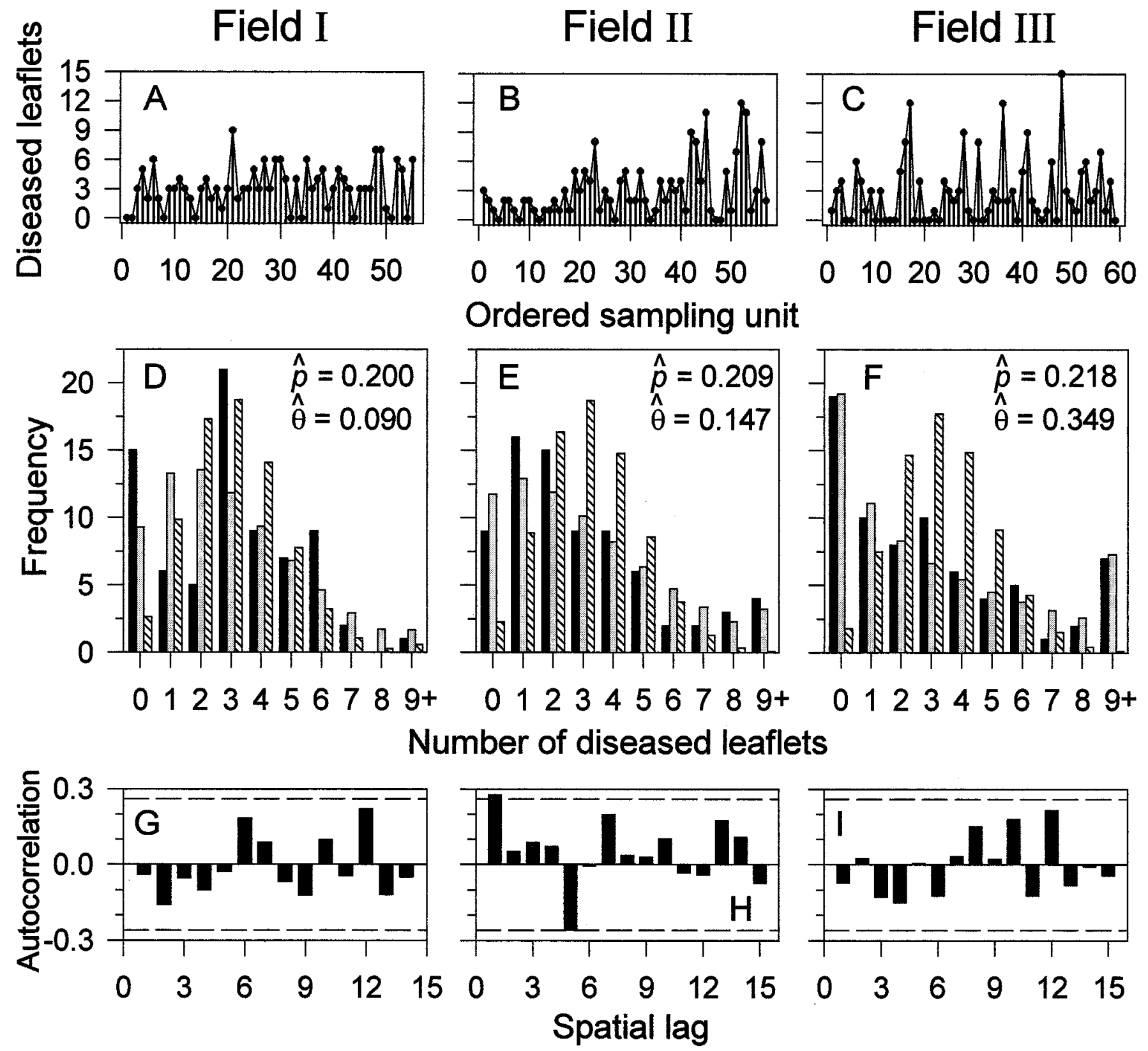

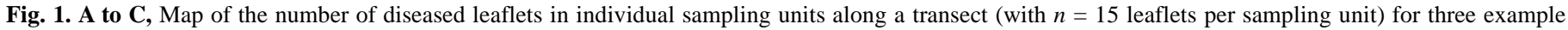

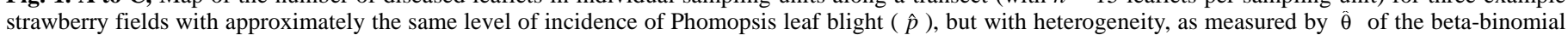

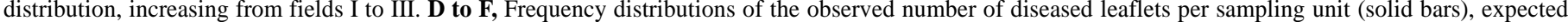

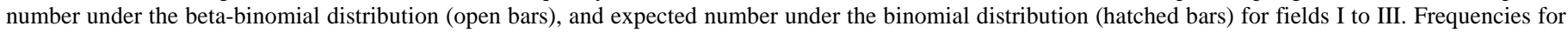

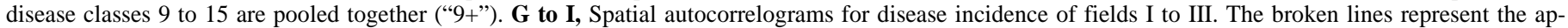
proximate $95 \%$ confidence intervals of the estimated autocorrelation coefficients. A, D, and G, Field I; B, E, and H, field II; and C, F, and I, field III. 

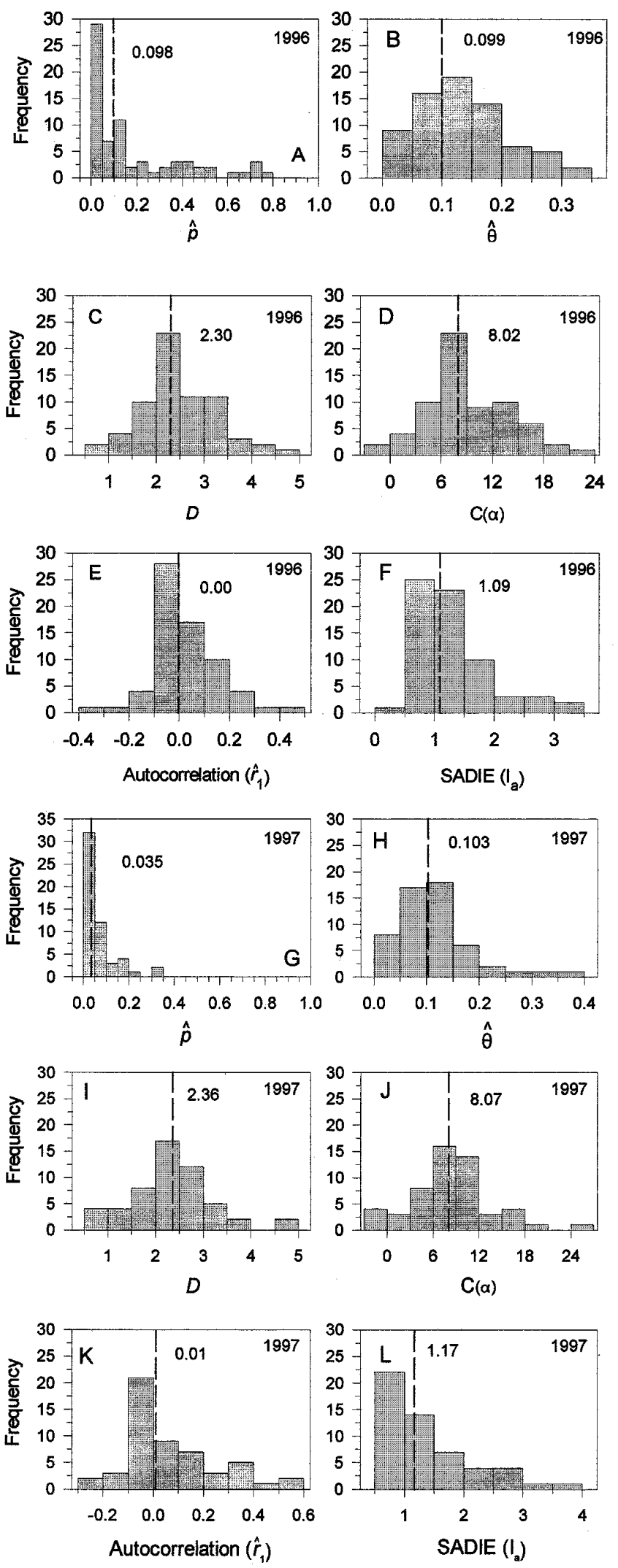

Fig. 2. Frequency distributions of the estimated beta-binomial parameters $\hat{p}$ and $\hat{\theta}$, the index of dispersion $(D)$, the standard normal statistic of the $C(\alpha)$ test $\left(z_{\mathrm{C}(\alpha)}\right)$, first-order spatial autocorrelation coefficient $\left(\hat{r}_{1}\right)$, and SADIE index of aggregation $\left(I_{\mathrm{a}}\right)$ for diseased leaflet data of $\mathbf{A}$ to $\mathbf{F}, 1996$ and $\mathbf{G}$ to $\mathbf{L}$, 1997. The frequency distributions are based on 67 and 54 data sets of the incidence of Phomopsis leaf blight at the leaflet level in 1996 and 1997, respectively. Vertical dashed lines represents the median value of the indicated statistic. Median is given numerically on the graph.
The $\chi^{2}$ test for $D(28)$, the normal approximation test of $D(46)$, and the $\mathrm{C}(\alpha)$ test of $D(28)$ all indicated significant heterogeneity in approximately $90 \%$ of the data sets assessed at the leaflet level in 1996 and 1997 (Table 1). The agreement in test results among these three tests of $D$ across all data sets was greater than $95 \%$, and results for $D$ were in high agreement with the LRS and goodness-of-fit tests for the beta-binomial and with the $t$ tests for $\hat{\theta}$ (Table 2). Heterogeneity of diseased leaves was not as high. This was indicated by a smaller percentage of significant results for the three tests of $D$ (Table 1), although there was high agreement among test results for leaves.

Binary power law. The binary power law provided an excellent description of the relationship between $v_{\text {obs }}$ and $v_{\text {ran }}$ on a log scale (Table 3, Fig. 3A to D). Estimated slopes and intercepts for the standard transect data sets were significantly $(P<0.01)$ greater than 1 and 0, respectively, for both 1996 and 1997 (Table 3). Thus, there was significant overdispersion of disease incidence across all the data sets. Most of the points were above the binomial line for the leaflet data (Fig. 3). Within a single spatial hierarchy (leaflet or leaf level), there was no significant difference between years in either the estimated slope or intercept, based on $t$ tests. Furthermore, a covariance analysis indicated that year did not affect the intercept or slope $(P>0.05)$. Estimates of $\ln \left(A_{x}\right)$ and $b$ were signifi-

TABLE 2. Percent agreement in the test results for the pairs of statistics used to assess heterogeneity of Phomopsis leaf blight of strawberry ${ }^{\mathrm{a}}$

\begin{tabular}{|c|c|c|c|c|c|}
\hline & \multicolumn{2}{|c|}{ Goodness of fit ${ }^{b}$} & \multirow[b]{2}{*}{ LRS $^{c}$} & \multirow[b]{2}{*}{$\theta^{\mathrm{d}}$} & \multirow[b]{2}{*}{$\chi^{2 \mathrm{e}}$} \\
\hline & BBD & BIN & & & \\
\hline BBD & * & $78.5(65)$ & $81.5(65)$ & $80.0(65)$ & $81.5(65)$ \\
\hline BIN & $57.6(33)$ & * & $3.8(78)$ & $9.2(78)$ & $3.8(78)$ \\
\hline LRS & $72.7(33)$ & $15.3(59)$ & $*$ & $91.1(112)$ & $98.3(121)$ \\
\hline$\theta$ & 72.7 (33) & $8.9(45)$ & 89.9 (79) & * & $85.7(112)$ \\
\hline$\chi^{2}$ & $69.7(33)$ & $20.3(59)$ & $90.9(121)$ & $81.0(79)$ & $*$ \\
\hline
\end{tabular}

${ }^{a}$ Data are pooled across 2 years. Upper triangle (above *) is for comparison of results at the leaflet level. Lower triangle (below *) is for comparisons at the leaf level. Except for the tests of goodness of fit, comparisons are based on agreement of two tests in rejecting the null hypothesis of randomness. The number of comparisons are in parentheses. Failure to reject the null hypothesis of a good fit for goodness-of-fit tests is compared with rejecting null hypothesis of the other tests.

${ }^{\mathrm{b}} \mathrm{BBD}=$ beta-binomial distribution; $\mathrm{BIN}=$ binomial distribution. The number of comparisons is lower for goodness-of-fit tests, because the test can only be performed when there are least three disease classes for BBD and two disease classes for BIN.

c The likelihood-ratio test statistic: two times the difference of the log-likelihoods for the beta-binomial and the binomial distributions. Significant results indicate that the beta-binomial provided a better fit than the binomial, and hence, the data exhibited overdispersion.

${ }^{d}$ Maximum likelihood estimate of the beta-binomial $\theta$ parameter. Significant departures from zero, determined with a $t$ test, indicate overdispersion.

${ }^{\text {e }} \chi^{2}$ test of index of dispersion, $D(28)$.

TABLE 3. Results of fitting the power law (28) (equation 2) to the incidence data of Phomopsis leaf blight of strawberry

\begin{tabular}{lccrc}
\hline Data set & $\hat{b}(\mathrm{SE})$ & $\ln \left(\hat{A}_{x}\right)(\mathrm{SE})$ & $\mathrm{df} \mathrm{f}^{\mathrm{b}}$ & $R^{2 \mathrm{c}}$ \\
\hline 1996 leaflets & $1.14(0.025)$ & $0.88(0.032)$ & 65 & 96.8 \\
1997 leaflets & $1.18(0.029)$ & $0.97(0.045)$ & 52 & 97.0 \\
1997 leaflets (intensive) $^{\mathrm{d}}$ & $1.12(0.047)$ & $0.89(0.084)$ & 13 & 97.6 \\
1997 leaflets (2D) & $1.11(0.065)$ & $0.91(0.074)$ & 6 & 97.6 \\
1996 leaves & $1.05(0.024)$ & $0.23(0.033)$ & 65 & 96.4 \\
1997 leaves & $1.09(0.023)$ & $0.29(0.042)$ & 52 & 97.7 \\
\hline
\end{tabular}

a $\hat{b}$ and $\ln \left(\hat{A}_{x}\right)$ are the estimated slope and intercept, respectively, of the best fitting line based on least squares regression. Number in parentheses are their standard errors.

${ }^{\mathrm{b}}$ Degrees of freedom for the regression.

${ }^{\mathrm{c}}$ Coefficient of determination (\%).

${ }^{\mathrm{d}}$ Additional sampling plans used only in 1997. Intensive = One-step spacing between sampling units instead of three steps. $2 \mathrm{D}=$ Sampling 10 sampling units in each of 10 parallel rows. 
cantly higher for disease incidence at the leaflet level than the leaf level (Table 3), based on $t$ tests. This may be due to the effect of sample-unit size (14), as indicated by the number of individuals in the sampling units ( $n=5$ or 15$)$ at the two hierarchical scales.
When disease data were pooled over the 2 years, estimates of $\ln \left(A_{x}\right)$ and $b$ were 0.91 (standard error $[\mathrm{SE}]=0.023)$ and $1.18(\mathrm{SE}=0.015)$, respectively, at the leaflet level; at the leaf level, estimates of $\ln \left(A_{x}\right)$ and $b$ were $0.25(\mathrm{SE}=0.023)$ and $1.07(\mathrm{SE}=0.014)$, respectively.
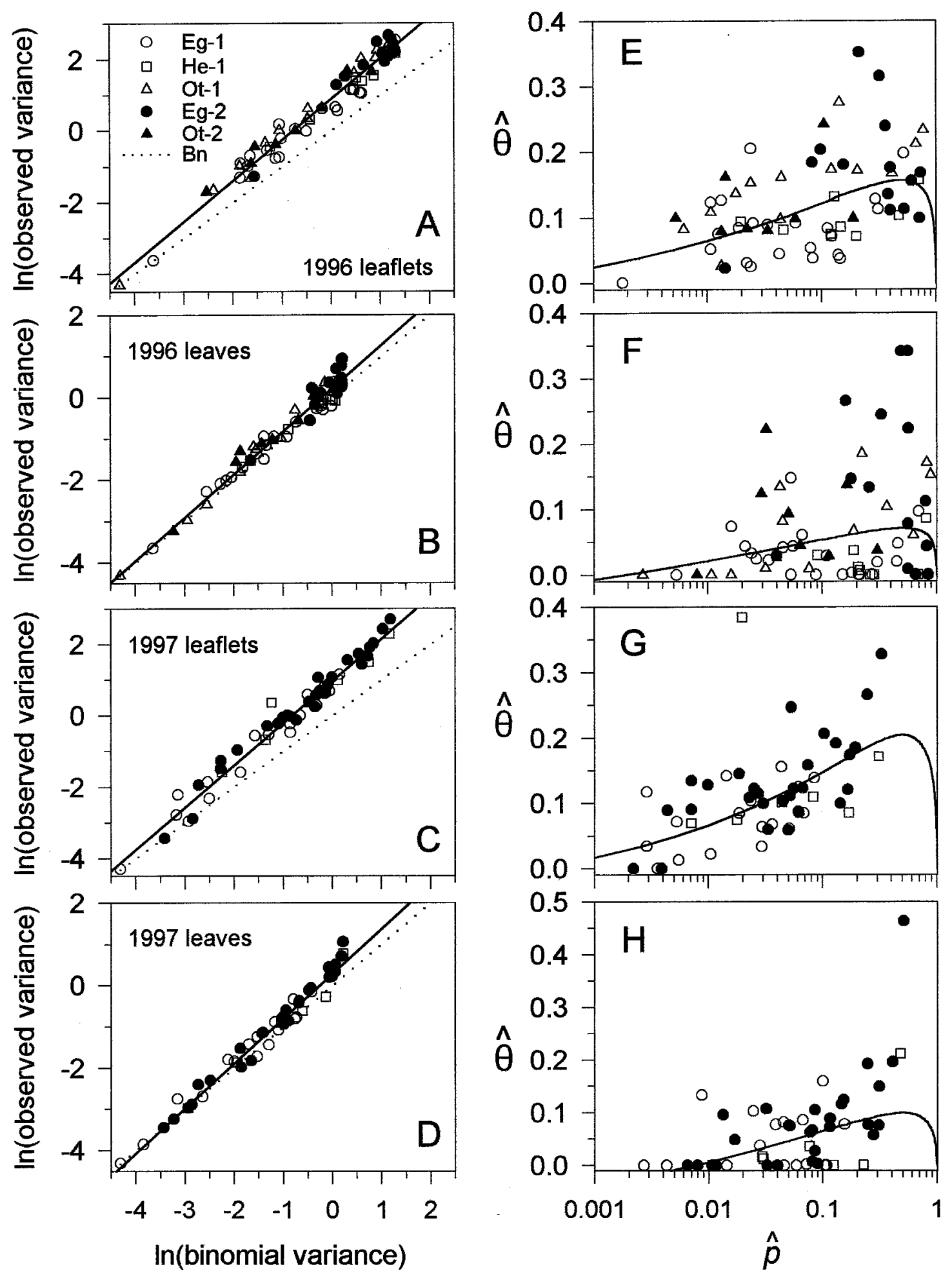

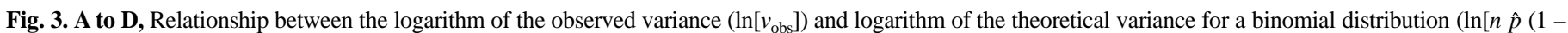

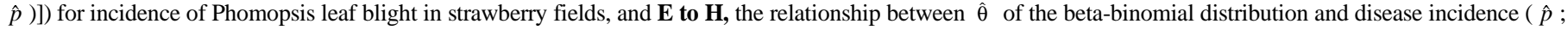

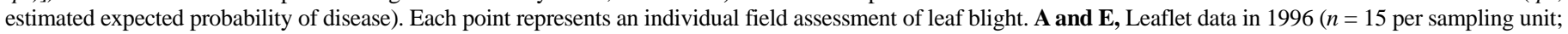

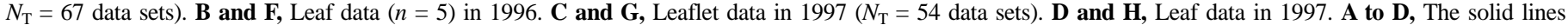

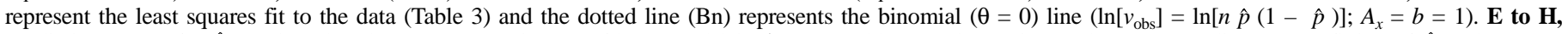

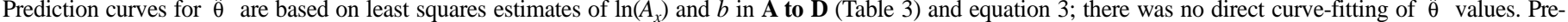

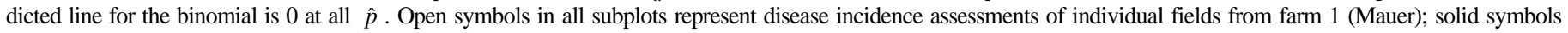

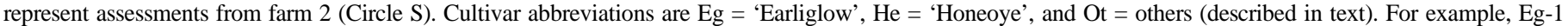
indicates 'Earliglow' at farm 1 . To maintain a consistent scale for $\hat{p}$, one low incidence value was omitted from $\mathbf{E}$ and $\mathbf{G}$; the values of $(\hat{p}, \hat{\theta})$ were $(0.0009,0.00)$. 
The effect of the factors farm, cultivar, and assessment date on the power law parameter estimates was determined from the results of the covariance analysis. Results varied somewhat by year. No factor significantly affected $b$ in either year (leaflet or leaf level); that is, no factor affected change in $\ln \left(v_{\text {obs }}\right)$ with change in $\ln (n p[1-p])$. Also, in 1997, no factor significantly affected $\ln \left(A_{x}\right)$; that is, no factor affected the height of the line in this year. In 1996, the cultivar factor significantly $(P<0.01)$ influenced $\ln \left(A_{x}\right)$ at the leaflet level. The 'other' cultivar level (all cultivars except 'Earliglow' and 'Honeoye') dominated this effect. The power law model can be rewritten to adjust for cultivar effects: $\ln \left(v_{\text {obs }}\right)=0.84+1.16 \cdot \ln \left(v_{\text {ran }}\right)-$ $0.10 \cdot \mathrm{He}+0.20 \cdot \mathrm{Ot}$, in which $\mathrm{He}=1$ if cultivar is 'Honeoye' and 0 otherwise, and Ot $=1$ if cultivar is 'other' and 0 otherwise. This gives estimated intercepts of $0.84,0.74$, and 1.04 for 'Earliglow', 'Honeoye', and 'other', respectively. The farm factor significantly $(P<$ 0.01) influenced the intercept estimate at the leaf level in 1996; farm 2 had a larger estimated $\ln \left(A_{x}\right)$ than farm 1 . As before, the power law model can be rewritten to adjust for farm effects at the leaf level: $\ln \left(v_{\text {obs }}\right)=0.15+1.03 \cdot \ln \left(v_{\text {ran }}\right)+0.18 \cdot \mathrm{F} 2$, in which F2 $=$ 1 if farm 2 is considered and 0 otherwise (if farm 1 is considered). This gives intercepts of 0.15 and 0.33 for farms 1 and 2, respectively. Thus, although there were significant changes in $\ln \left(A_{x}\right)$ with respect to these factors, their influence on $\ln \left(v_{\text {obs }}\right)$ was extremely small.
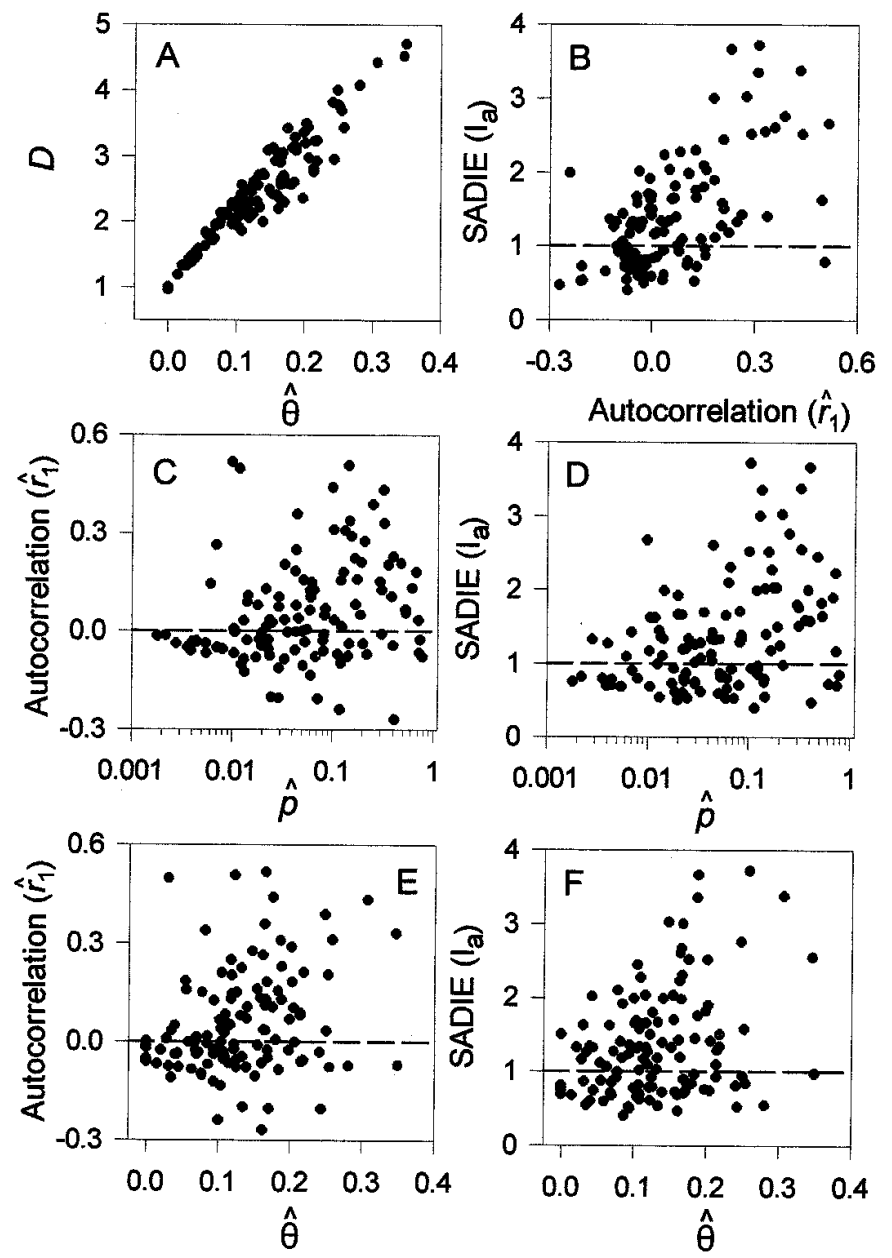

Fig. 4. Bivariate relationships between $\mathbf{A}$, estimated beta-binomial parameter $\hat{\theta}$ and index of dispersion $(D)$; $\mathbf{B}$, first-order spatial autocorrelation coefficient $\left(\hat{r}_{1}\right)$ and SADIE's index of aggregation $\left(I_{\mathrm{a}}\right)$; $\mathbf{C}$, beta-binomial $\hat{p}$ parameter and $\hat{r}_{1} ; \mathbf{D}$, beta-binomial $\hat{p}$ parameter and $I_{\mathrm{a}} ; \mathbf{E}$, beta-binomial $\hat{\theta}$ parameter and $\hat{r}_{1}$; and $\mathbf{F}$, beta-binomial $\hat{\theta}$ parameter and $I_{\mathrm{a}}$. Each point represents a single field assessment of the incidence of Phomopsis leaf blight of strawberry at the leaflet level (e.g., $\hat{p}$ is the estimated expected probability of a leaflet being diseased). Data from 1996 and 1997 are pooled $\left(N_{\mathrm{T}}=121\right)$.
The binary power law parameter estimates were used to develop prediction curves for the beta-binomial aggregation parameter, $\theta$, based on equation 3 for the individual years (Fig. 3E to $\mathrm{H}$ ). Thus, the curves in Figure $3 \mathrm{E}$ to $\mathrm{H}$ were not based on direct curve-fitting of the $\hat{\theta}$ values. Although, the predicted $\hat{\theta}$ values based on equation 3 followed the general trend of $\hat{\theta}$ with $\hat{p}$ (Fig. $3 \mathrm{E}$ to $\mathrm{H}$ ), there was considerable variation in the individual $\hat{\theta}$ values at any given $\hat{p}$ for both leaflets and leaves. In general, $\hat{\theta}$ increased with $\hat{p}$ until $\hat{p} \approx 0.5$ and then decreased at higher $\hat{p}$ levels. There was less of a systematic change in $\hat{\theta}$ with $\hat{p}$ for leaves than for leaflets because $\hat{b}$ was lower for leaves (Table 3$)$; at $\hat{b}=1\left(A_{x}>1\right)$, predicted $\theta$ from equation 1 is a horizontal line. Similar plots were obtained for other measures of heterogeneity such as $D$ in relation to $\hat{p}$; these are not shown here because of the high agreement in results among the various indices (Table 2). For instance, the linear relationship between $\hat{\theta}$ and $D$ is shown in Figure 4A. The correlation coefficients between the individual $\hat{\theta}$ values and those predicted by equation 3 were all significant $(P<0.05)$ but very low.
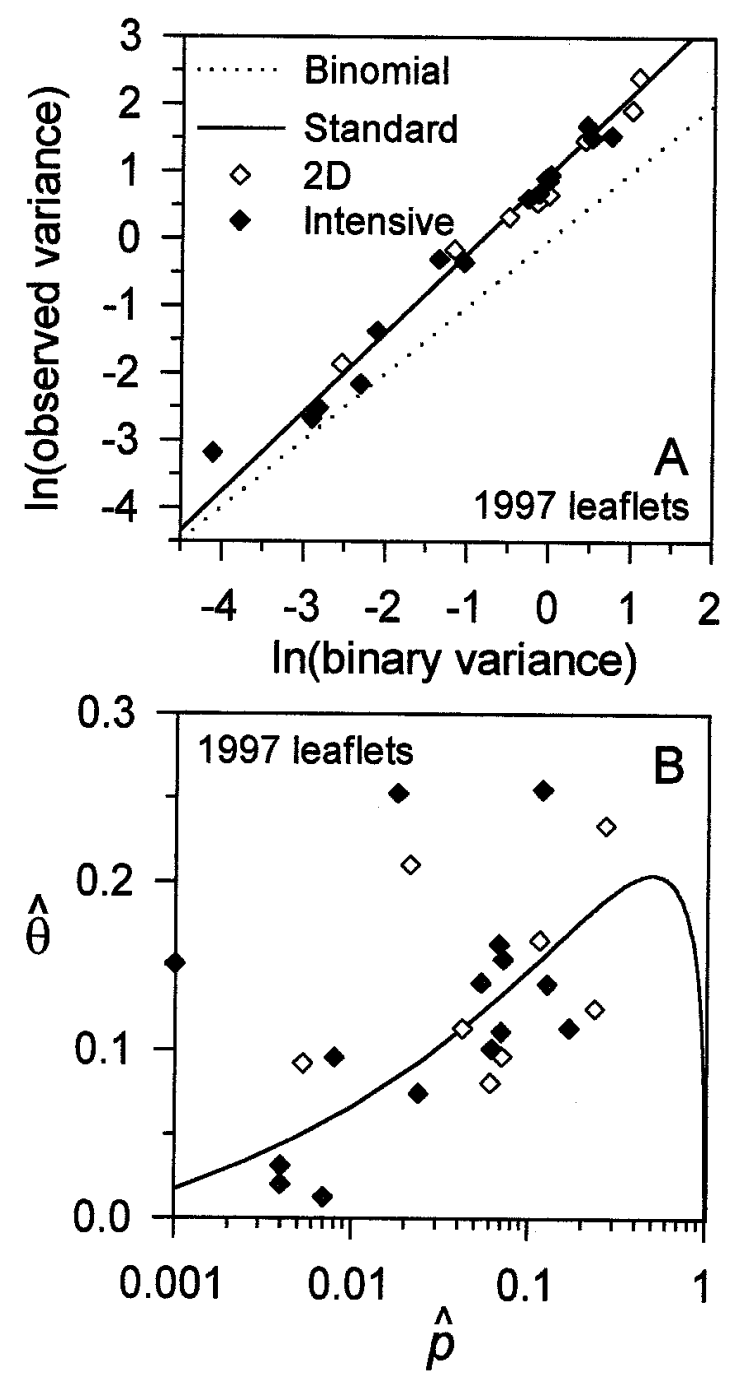

Fig. 5. A, Relationship between the logarithm of the observed variance $\left(\ln \left[v_{\mathrm{obs}}\right]\right)$ and $\operatorname{logarithm}$ of the theoretical variance for a binomial distribution $(\ln [n \hat{p}(1-\hat{p})])$ for the incidence of Phomopsis leaf blight in strawberry fields. Data are for the two-dimensional (2D; open diamonds) and intensive transect (solid diamonds) sampling plans for diseased leaflet data in 1997. Disease incidence is represented by $\hat{p}$ (estimated expected probability of disease). Solid line represents the power law fit to the diseased leaflet data in Figure $3 \mathrm{C}(0.97+1.18 \cdot \ln [n \hat{p}(1-\hat{p})])$ (Table 3$)$ and not a fit to the data in this figure. The dotted line represents the binomial line $\left(\ln \left[v_{\text {obs }}\right]=\ln [n \hat{p}(1-\hat{p})]\right)$. $\mathbf{B}$, Estimates of $\hat{\theta}$ for $2 \mathrm{D}$ and intensive sampling plans. Solid line curve is $\hat{\theta}$ based on equation 3 using estimated parameters of power law results fitted to the leaflet data in Figure 3C. 
There was not an exact relationship between $\hat{\theta}$ and $\hat{p}$, because the fit of the binary power law to the variance was not exact $\left(R^{2}<\right.$ 1.0 ). Even though $R^{2}$ values were very high (Table 3 ), the small deviations from the best fitting lines (Fig. 3A to D) were in log units. A small difference between the observed and predicted logs of variance could translate into a large difference in $\hat{\theta}$ values predicted with equation 3 .

The observed variance data $\left(v_{\mathrm{obs}}\right)$ of the intensive transect samplings and the 2D sampling plans of 1997 were in very good agreement with the power law results (Fig. 5A). Data points were very close to the prediction values determined from the standard transect samplings (Fig. 5A; solid line). Individual $\hat{\theta}$ values generally increased with $\hat{p}$ for the intensive samplings and 2D sampling plans (Fig. 5B), and there was also a high degree of variability of $\hat{\theta}$ at any $\hat{p}$. Predicted values from equation 3 (with parameters determined from the standard transect) (Table 3) showed the same trend as the individual $\hat{\theta}$ s. Estimates of $\ln \left(A_{x}\right)$ and $b$ (Table 3 ) were very similar to those from the standard transects.

Correlation analysis. Spatial autocorrelation. First-order autocorrelation coefficients $\left(\hat{r}_{1}\right)$ for diseased leaflets ranged from -0.27 to 0.52 (Fig. 2E and $\mathrm{K}$ ), with a median of 0 in each of the 2 years. Autocorrelograms are shown in Figure $1 \mathrm{G}$ to I for the three fields. Only in field II (Fig. 1H) was there significant $(P<0.05)$ autocorrelation (Table 1$)$. First-order coefficients $\left(\hat{r}_{1}\right)$ were almost always small, with less than $20 \%$ of the data sets exhibiting significant $(P<$ 0.05) $\hat{r}_{1}$ values (Table 1). The percentage of data sets with $\hat{r}_{1}$ significantly different from 0 was somewhat smaller in 1996 than in 1997 (Table 1). Results were very similar for leaflets and leaves (Tables 1 and 4). There was no general evidence of a particular type of autoregressive or moving-average process (43) for the disease incidence data (Fig. $1 \mathrm{G}$ to I), even when there was identified spatial autocorrelation.

The magnitude and variability of $\hat{r}_{1}$ values were related to $\hat{p}$ (Fig. 4C). Most values of $\hat{r}_{1}$ were close to 0 at $\hat{p}<0.01$. Above $1 \%$ disease incidence, $\hat{r}_{1}$ values and the variation in $\hat{r}_{1}$ tended to increase with $\hat{p}$. A covariance analysis relating $1 / \hat{r}_{1}$ to $\log (\hat{p})$ and the factors year, farm, cultivar, and assessment date did not show a significant effect of the factors on the magnitude of the first-order autocorrelation $(P>0.05)$. There was also an increase in the magnitude of $\hat{r}_{1}$ and variability in $\hat{r}_{1}$ with increasing $\hat{\theta}$ of the beta-binomial distribution (Fig. 4E).

Autocorrelation analysis of the more intensively sampled transects yielded results similar to those of the standard transect samples. Significant first-order autocorrelations were found more frequently for the intensive samples $(27 \%)$ than the standard transect samplings (17\%) (Table 1) for diseased leaflets in 1997, but this may be a function of $N$, since the magnitude of the $\hat{r}_{1}$ was similar for the two sampling approaches. For the 14 intensively sampled transects, $\hat{r}_{1}$ ranged from -0.17 to 0.36 , with a median of 0.07 . Because the sampling units of the intensive transects were positioned one step apart, we were able to extract three subsets from these transects to compare results for one-step separation with three different three-step (standard) separations of sampling units. Estimates of $\hat{r}_{1}$ for the three subtransects were either larger, smaller, or the same as $\hat{r}_{1}$ for the full (intensive) transects. For the $3 \times 14$ subtransects, $\hat{r}_{1}$ ranged from -0.20 to 0.52 , with a median of 0.07 . These results are similar to those obtained from the intensively sampled transects, indicating that our standard three-step sampling scheme did not allow any significant spatial correlation among sampling units to go undetected. Moreover, $\hat{p}$ was not affected by the subtransect samplings (i.e., the median, minimum, and maximum of the data were nearly identical). Spatial autocorrelation coefficients for the 2D samples were also low. For the eight fields sampled in this manner, $\hat{r}_{1}$ ('rook' case) ranged from -0.12 to 0.38 , with a median of $0.11 ; \hat{p}$ ranged from 0.0053 to 0.27 . These results are very similar to the standard transect results (Fig. $2 \mathrm{G}$ and $\mathrm{K}$ ). In

TABLE 4. Percent agreement in the test results for the pairs of statistics used to assess spatial associations of Phomopsis leaf blight of strawberry ${ }^{\mathrm{a}}$

\begin{tabular}{lcccc}
\hline & & \multicolumn{2}{c}{ Runs $^{\mathrm{c}}$} \\
\cline { 3 - 4 } & $r_{1}{ }^{\mathrm{b}}$ & Median & Ordinary & SADIE $^{\mathrm{d}}$ \\
\hline$r_{1}$ & $*$ & $86.7(120)$ & $86.5(111)$ & $84.2(120)$ \\
Median & $86.7(120)$ & $*$ & $90.1(111)$ & $80.8(120)$ \\
Ordinary & $87.4(111)$ & $91.9(111)$ & $*$ & $74.8(111)$ \\
SADIE & $83.3(120)$ & $78.3(120)$ & $74.8(111)$ & $*$ \\
\hline
\end{tabular}

a Data are pooled across 2 years. Upper triangle (above *) is for comparison of results at the leaflet level. Lower triangle (below $*$ ) is for comparisons at the leaf level. The number of comparisons are in parentheses.

b The first-order autocorrelation coefficient, $r_{1}$, indicates significant spatial correlation in disease incidence in neighboring sampling units.

${ }^{c}$ Median and ordinary runs analyses indicate significant associations in disease status in neighboring sampling units. Ordinary runs: each sampling unit is classified as disease free (0) or diseased (1; one or more diseased leaflets) (10). Median runs: each sampling unit is classified as being below (0) or above (1) the median incidence.

${ }^{d}$ SADIE (38) is based on the number of moves to regularity of the observed data $\left(D_{\mathrm{a}}\right)$ relative to the moves to regularity for randomizations of the observed data. $P_{\mathrm{a}}$ is the proportion of SADIE randomizations that are larger than $D_{\mathrm{a}}$ and was used to determine the significance ( $P$ value) of a two-sided hypothesis test of nonrandomness. Significance for this test was determined from $2 \cdot \min \left(P_{\mathrm{a}}, 1-P_{\mathrm{a}}\right)$, in which $\min (\bullet, \bullet)$ is the 'minimum' function.

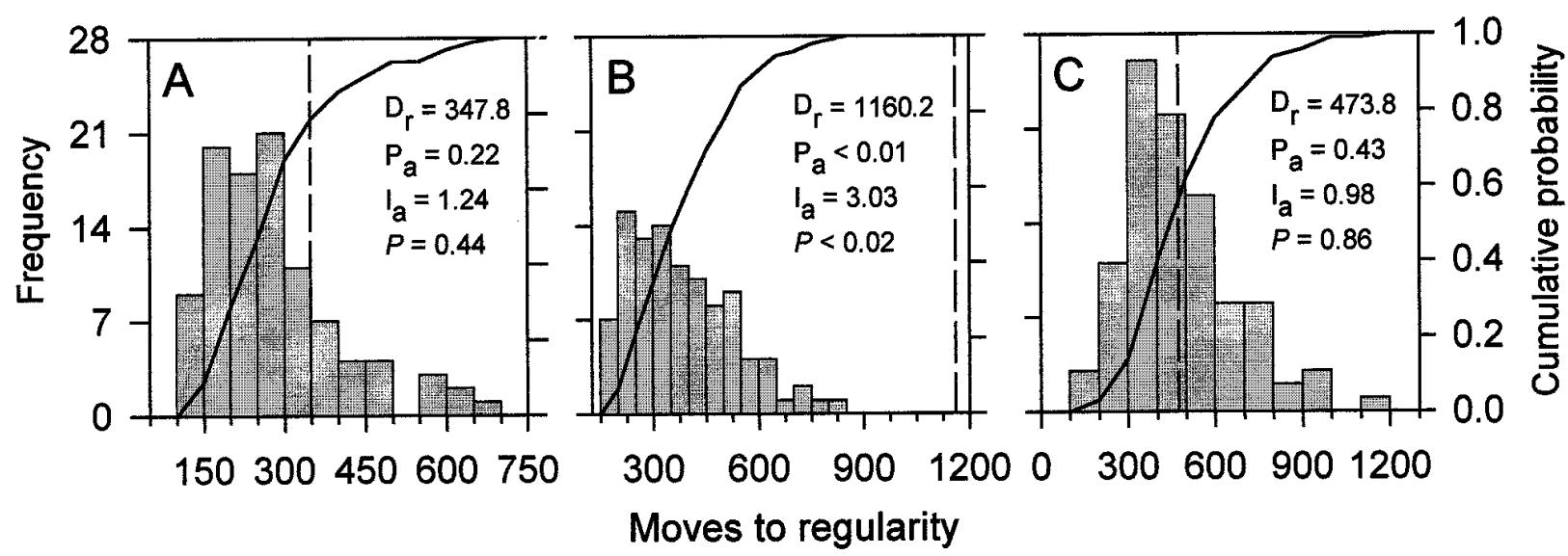

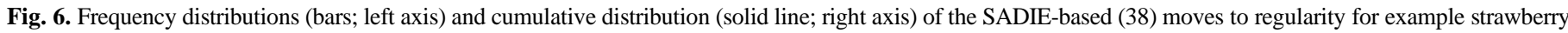

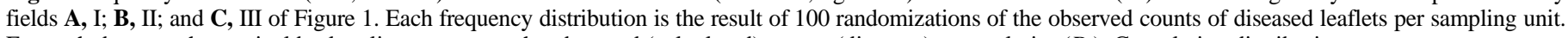

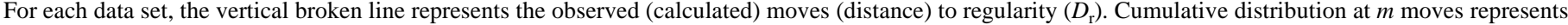

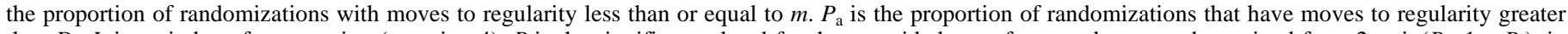

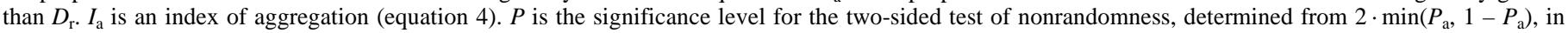
which $\min (\bullet, \bullet)$ is the 'minimum' function. 
only one $2 \mathrm{D}$ data set was $\hat{r}_{1}$ significantly greater than $0(P<0.05)$. Other proximity measures ('row' and 'column') gave nearly identical results (data not shown).

Runs analysis. Median and ordinary runs analyses were able to detect significant $(P<0.05)$ nonrandom patterns of disease incidence in 9 to $17 \%$ of the standard transects over the 2-year study (Table 1). The percentages were slightly higher for 1997 than for 1996, but the results for leaflets and leaves were similar. Ordinary runs analysis, unlike median runs, must give the same results regardless of which spatial hierarchy was used, because the sampling units have the same disease status whether or not leaves or leaflets are used as the basis of disease incidence. The agreement in significant results between ordinary and median runs was greater than $90 \%$ at both the leaflet and leaf levels (Table 4). Moreover, agreement between first-order spatial autocorrelations and both runs tests was $>83 \%$ (Table 4 ).

SADIE. For all data sets, the SADIE program was able to calculate distance to regularity $\left(D_{\mathrm{r}}\right)$ and the distribution of the distance to regularity for randomized arrangements of the samplingunit counts. In general, SADIE was able to detect departures from randomness more often than both spatial autocorrelation or runs analyses (Table 1 ), with 16 to $23 \%$ of the cases being significantly nonrandom $(P<0.05)$. The percentages were higher in 1997 than in 1996, and leaflets and leaves had about the same results (Table 1). Frequency distributions of the SADIE index, $I_{\mathrm{a}}$, across all data sets were positively skewed (Fig. $2 \mathrm{~F}$ and L). The skewness was, in part, due to the lower bound of 0 imposed on $I_{\mathrm{a}}$. The distribution of $I_{\mathrm{a}}$ values had median values of 1.1 and 1.2 at the leaflet level in 1996 and 1997, respectively.

Randomization results of SADIE from the three fields in Figure 1 are shown in Figure 6. Frequency distributions of these distances to regularity were all positively skewed. For field II, the calculated distance ("moves") to regularity ( $D_{\mathrm{r}}$, vertical broken line) was greater than all distances to regularity for the randomized placement of counts per sampling unit. This resulted in a value of $P_{\mathrm{a}}<$ 0.01 , the smallest possible value for the number of simulations conducted (100). For field III, $D_{\mathrm{r}}$ was greater than $43 \%$ of all the distances to regularity of the randomized counts, thus giving a $P$ value of 0.86 .

The percent agreement in significant results between SADIE and the other spatial statistics (spatial autocorrelation and runs analyses) was around $80 \%$, slightly lower than the agreement in significant results between spatial autocorrelation and runs analyses (Table 4). The lower rate of agreement was because more data sets had a significant $I_{\mathrm{a}}$ than $\hat{r}_{1}$ (Table 1). The agreement in the test results can be seen by the generally increasing $I_{\mathrm{a}}$ with increasing $\hat{r}_{1}$ (Fig. 4B).

Values of $I_{\mathrm{a}}$ and the variability of $I_{\mathrm{a}}$ generally increased with $\hat{p}$ (Fig. 4D). Although there is no specific critical value of $I_{\mathrm{a}}$ for significant nonrandomness (36), most of the significant SADIE results were associated with $\hat{p}>0.10$. Even though there was no expectation that there be a relationship between $\hat{\theta}$ and $I_{\mathrm{a}}$, there was a general increase in $I_{\mathrm{a}}$ with $\hat{\theta}$ (Fig. $\left.4 \mathrm{~F}\right)$.

SADIE results did not change much for the $2 \mathrm{D}$ samplings in that results were not significant in seven of the eight data sets. Moreover, SADIE results were only slightly different for the intensive samplings. In 6 of the 14 data sets, $I_{\mathrm{a}}$ was larger for the intensive sample than the three subsets of more widely spaced transects. In four other data sets, one of the subsets had a larger $I_{\mathrm{a}}$ than the $I_{\mathrm{a}}$ of the intensive sample. In the other cases, $I_{\mathrm{a}}$ for the intensive sample was similar to the values for the subsets.

\section{DISCUSSION}

Spatial pattern is a useful ecological characteristic that helps define a population such as diseased strawberry leaflets $(1,9,41)$. However, few researchers have drawn upon this "virtually untapped reservoir of potentially useful information" (9). In this study, the spatial pattern of Phomopsis leaf blight of strawberry in perennial production systems was characterized over 2 years using a collection of statistical methods that measure the heterogeneity (overdispersion) of disease incidence and the spatial arrangement of disease incidence. In particular, the beta-binomial distribution mostly provided a good fit to the data for diseased leaflets and leaves, and the estimated aggregation parameter of this distribution $(\theta)$, as well as closely related indices (e.g., $D$ ), indicated that there was significant heterogeneity in most data sets. Results based on the binary power law indicated that the degree of heterogeneity depended primarily on magnitude of disease incidence and only inconsistently with other factors. However, statistical measures of spatial correlation, including the recently developed SADIE approach, indicated that in most data sets there was little evidence of nonrandom spatial arrangement of diseased individuals per sampling unit over the area of the fields sampled. In about $20 \%$ or fewer of the data sets, there was evidence of relatively small-magnitude patterns to the arrangement of disease incidence. A fuller evaluation of the meaning of the statistical tests used here in relation to the disease-host system and method of sampling can reveal a considerable portion of the nature of the spatial pattern of strawberry leaf blight.

Unless the pattern of disease incidence is purely random (i.e., every leaflet has the same probability of being diseased), there can be various types and magnitudes of manifested patterns, depending upon the association of diseased individuals with specific locations and with other diseased individuals various distances away $(14,26)$. Because of this scale dependence, more than one method is typically required to characterize disease patterns when the data consist of counts of diseased individuals in sampling units with known locations (26). The two methodologies used here (pointpattern and correlation-type analyses) identify different aspects of pattern of Phomopsis blight, and the results should be used collectively to describe pattern of disease incidence.

The arrangement of organisms in space is believed to be dependent upon a variety of scale-specific physical, chemical, and biological processes (14). Complicating the situation is that the process-driven scale dependence can be confounded with measurement-based scale dependence (e.g., if sampling-unit size is arbitrary) (14,22). With the Phomopsis leaf blight data, heterogeneity was affected by number of plant units (15 leaflets or 5 leaves) per sampling unit (Table 1). However, spatial associations in disease incidence between sampling units (measures of correlation) were not noticeably affected by whether leaflets or leaves were used to determine the disease status of the sampling unit (Tables 1 and 4). The remainder of this discussion primarily focuses on the pattern of disease at a single plant-unit level, the leaflets.

We were able to show that characterizing the pattern of disease incidence could be accomplished using simple data-collection methods such as the use of one-dimensional transects. Results from the intensively sampled transects and the 2D samples were very similar to the results from the standard transects of fields (Fig. 5). Most likely this is because randomly chosen transects through the commercial fields were capturing the essential features of the dispersion of diseased leaflets.

The beta-binomial discrete distribution provided a good fit (based on a $\chi^{2}$ goodness-of-fit test) to most Phomopsis data sets and a better fit to the data than the binomial in over $80 \%$ of the cases. The relatively good fit of the binomial to the leaf data reflects the lower degree of heterogeneity at this spatial scale. A direct measure of the heterogeneity of disease incidence or, in the terminology of Pielou (41), the "intensity" of pattern was obtained with the estimated $\theta$ parameter of the beta-binomial, $\hat{\theta}$, which ranged greatly among the data sets, although magnitude of the estimates was similar to that found in other studies $(29,31,47)$. This parameter can be reformulated as $\rho=\theta /(1+\theta)$, the intracluster correlation, and is a measure of the degree of association of the disease status of leaflets (or leaves) within sampling units. In this context, one can see that the point-pattern distributional approach, although based 
on variation in counts of diseased leaflets (or leaves) among sampling units, is a direct measure of association of disease status within sampling units. High heterogeneity (determined by $\hat{\theta}$ ) means high similarity of disease status within the units. In other words, the distributional approach assesses the pattern of disease at or below the spatial scale of the sampling unit $(2,26)$.

Various statistics can be used to assess the degree of nonrandomness of disease incidence at the level of the sampling unit (28). In this study, the beta-binomial $\theta$ parameter, LRS, the index of dispersion $(D)$, the standard normal statistics of the $\mathrm{C}(\alpha)$ test $\left(z_{\mathrm{C}(\alpha)}\right)$, and the Sun and Madden test $\left(z_{\mathrm{sm}}\right)$ were all used to assess the degree of nonrandomness. Tables 1 and 2 show the very high agreement among these various statistics. There was a high degree of agreement (redundancy) among these indices under the conditions of our study, thus, it is necessary to briefly compare these measures of nonrandomness.

The $z_{\mathrm{C}(\alpha)}, z_{\mathrm{sm}}$, and $\chi^{2}$ test statistics are all functions of $D(28,46)$. There is a direct relationship between $z_{\mathrm{C}(\alpha)}$ and $z_{\mathrm{sm}}$ that can be written as $z_{\mathrm{C}(\alpha)}=z_{\mathrm{sm}}[(N-1) / N]^{1 / 2}-[2 N(1-1 / n)]^{-1 / 2}$. For $n=15$ and $N=$ $75, z_{\mathrm{C}(\alpha)}=0.99 \cdot z_{\mathrm{sm}}-0.084$, virtually the same as $z_{\mathrm{sm}}$. Furthermore, Sun and Madden (46) have shown that the $\chi^{2}$ is more conservative than the standard normal test $\left(z_{\mathrm{sm}}\right)$; however, the difference between them is quite small when $n>10$ and $N>50$, as found here for leaf blight of strawberry (Table 1). When the beta-binomial describes the frequency distribution of diseased individuals, as with our data, it may be preferable to quantify aggregation in terms of $\theta$ and test heterogeneity using a $t$ test based on the maximum likelihood-derived standard error of $\hat{\theta}$. The $t$ test for $\hat{\theta}$ gave results very similar to the tests based only on variances (Table 1 ), and there was a high degree of correlation between the magnitude of $\hat{\theta}$ and the other indices (Table 2, Fig. 4A). Such a high agreement in the test results may also be a function of $n, N$, and the acceptability of the beta-binomial for representing disease distribution. With the values of $n$ and $N$ used in this study, there was little advantage in the maximum likelihood estimation in terms of testing for overdispersion. If one did not have access to BBD or other specialized software, one could use the moment estimates of $\theta$ or $\rho$ (e.g., $\hat{\rho}=$ $[D-1] /[n-1])$ because the moment estimates typically are similar to the maximum likelihood estimates. All tests of overdispersion would then be strictly based on $D$ (and, hence, variances). If one wanted to statistically compare nonzero $\hat{\theta}$ values from two different data sets for equality or test for the equality of $\theta$ to a nonzero value, then maximum likelihood estimates would be of great benefit, because the estimated asymptotic standard error of $\hat{\theta}$ can be used in $t$ tests involving $\hat{\theta}$ values (28). To do the same thing with the other indices, estimates of their standard errors would have to be obtained using resampling procedures (e.g., the jackknife [42]).

Justification for the beta-binomial distribution is strengthened for our study by the excellent fit of the binary power law $(18,28-31)$ to the Phomopsis leaf blight data (Fig. 3A to D). Power law results were very consistent between years and showed that the degree of heterogeneity was a function of $\hat{p}$. Hughes and Madden (17) previously showed the link between the power law parameters and the moments of the beta-binomial distribution, resulting in a prediction equation for $\theta$ based on $\hat{p}, \hat{A}_{x}$, and $\hat{b}$ (equation 3). This equation indicates that $\theta$ increases to a maximum (at $p=0.5$ ) and then declines (Fig. $3 \mathrm{E}$ to $\mathrm{H}$ ) when $\hat{b}>1$. Estimates of $\theta$ generally followed this trend, but the $\hat{\theta} \mathrm{s}$ varied considerably at any given $\hat{p}$. This high variability of $\hat{\theta}$ in relation to $\hat{p}$ was found by others $(31,34)$. Thus, although degree of heterogeneity of Phomopsis leaf blight was, on average, related to mean disease incidence (with greatest heterogeneity around $50 \%$ incidence), individual data sets had a range of heterogeneity levels that could be attributed to other causes. There was less of a change in $\hat{\theta}$ with increasing $\hat{p}$ for leaves than for leaflets, but this is consistent with the lower $\hat{b}$ for leaves (Table 3) than for leaflets.

The good fit of the binary power law to the variance data was exploited in a covariance analysis to test for factors that signifi- cantly affected estimates of the intercept $\left(\ln \left[A_{x}\right]\right)$ and slope $(b)$ of equation 1 and, thus, the degree of heterogeneity (and degree of intracluster correlation) of disease incidence (29). In 1996 but not in 1997, we found that the power law results were affected by cultivar at the leaflet level and by farm location at the leaf level. These factors only changed the intercept $\left(\ln \left[A_{x}\right]\right)$, suggesting that the degree of heterogeneity (and, hence, the correlation of disease status within sampling units) was consistently changed by cultivar or farm at all levels of $\hat{p}$. There are two likely reasons why the factors that influenced the power law results in 1996 did not influence the relationship in 1997. First, the cultivars grouped together as 'other' were not assessed in 1997, and this category had the largest effect in 1996. Second, disease incidence was lower in 1997, and values of $\hat{p}$ over 0.5 may be needed to detect the effect of a factor.

For the binary power law, accounting for significant factors only marginally improved the fit and only slightly changed the height of the lines. It should be pointed out that lack of an effect of a factor (e.g., assessment date) on power law results did not mean that the factor was not having an influence on disease heterogeneity. For instance, assessment date did influence $\hat{p}$ (data not shown), and $\hat{p}$ clearly influenced $\ln \left(v_{\text {obs }}\right)$ (Fig. 3A to D) and $\hat{\theta}$ (Fig. 3E to H). However, after correcting for the effect of $\hat{p}$ on heterogeneity, assessment date (or other nonsignificant factors) did not account for any more of the variability in $\ln \left(v_{\mathrm{obs}}\right)$.

When individuals are intensively mapped, there are various pointpattern methods for characterizing aggregation at multiple scales $(4,23,26,45)$. As an alternative, spatial correlation-type analyses (including runs analysis) can be performed on both intensively mapped (not applicable in this study) or sparsely sampled individual data to characterize pattern at the sampling-unit level and above (26,28,31,35), as long as the (relative) positions of the sampling units are known. These analyses characterize what Pielou (41) termed as the "grain" of the spatial pattern, in contrast to the intensity of the pattern (measured with $\hat{\theta}$ and related statistics). Characters of grain include the (average) size of the clusters of disease, as well as the positions of these clusters relative to each other.

In general, spatial correlation coefficients for Phomopsis leaf blight were low at both the leaflet and leaf levels for both years (Fig. $2 \mathrm{~K}$ and E). In the approximate $12 \%$ of the fields that showed significant first-order autocorrelations, $\hat{r}_{1}$ values were small $\left(\left|\hat{r}_{1}\right|<\right.$ 0.5 , in general) and autocorrelograms did not give any indication of a particular autoregressive or moving-average (ARIMA) process. About the same percentage of data sets showed spatial patterns using the two different runs analyses (Table 1). Similar results were found for other diseases (31-33). However, in some studies (13, $15)$, higher first-order correlations were detectable and, sometimes, the data could be modeled as an ARIMA process $(15,43,44)$.

The new SADIE approach (36-38) tested here is an alternative to autocorrelation and runs analyses that is specifically conditioned on heterogeneity of the data set. This conditioning is manifested in the randomization test; i.e., each random rearrangement of the sampling-unit counts has the same level of heterogeneity. In other words, the variance of the counts of diseased leaflets, the frequency distribution of the counts in the sample, and the indices of aggregation remain unchanged. Using SADIE, results were similar to those for spatial autocorrelation and runs analyses (Tables 1 and 4), although the null hypothesis (of a random arrangement) was rejected more frequently with SADIE than for the other tests. This may reflect the fact that, because SADIE is based on randomizations of the observed data rather than on assumptions about the statistical distribution of the test statistic (e.g., standard normal for runs), this new procedure was somewhat more powerful than the other tests (with our data) for detecting nonrandomness. The large values of $I_{\mathrm{a}}$ from the SADIE analysis were associated with large values of $\hat{r}_{1}$ (Fig. 4B). This suggests that the level of heterogeneity in the data sets was not having a great influence on the correlation-type analyses of Phomopsis leaf blight, probably because 
heterogeneity was not large (Fig. 2C and I) compared with that found in some other studies (28).

The three fields of Figure 1 can be used to demonstrate the combined meaning of the statistical results. All three fields had the same $\hat{p}$, but heterogeneity increased from I to III, meaning that correlation of disease status of the leaflets within the sampling units increased from field I to III (Fig. 1A to C). Only field II had significant positive spatial association of disease incidence among sampling units, as assessed by $\hat{r}_{1}$ (Fig. $\left.1 \mathrm{H}\right)$ or $I_{\mathrm{a}}$ of SADIE (Fig. 6B). In fact, for the SADIE randomizations, no rearrangement of the counts for this field produced a larger distance to regularity than the observed $D_{\mathrm{r}}$. The values of $\hat{r}_{1}$ for this field was only moderate in magnitude, but was typical for those data sets with significant correlation coefficients (Fig. 4C). The map and statistics for field II are indicative of similar levels of disease incidence occurring together, or equivalently, clusters of disease extending beyond the borders of the sampling units. On the other hand, field III had the greatest degree of heterogeneity of the three shown (Fig. 1C), but had a nearly random arrangement of sampling units $\left(I_{\mathrm{a}} \approx 1\right.$, Fig. 6C; $\hat{r}_{1} \approx 0$, Fig. 1I). The maps and statistical results for both fields I and III are indicative of small clusters of diseased leaflets, roughly the size of the sampling units or smaller. In other words, there was no visible evidence of sections of the transects where disease incidence was mostly high or low. Of these two fields, the difference between the high incidence and low incidence sampling units was much greater in field III than in field I. This can also be seen by the frequency distribution of diseased leaflets (Fig. 1D and F). Field III had a relatively large number of sampling units with zero or nine diseased leaflets; field I had few sampling units with nine diseased leaflets but many with an intermediate number of diseased leaflets, which is a reflection of a low level of heterogeneity. The complexity of the differences between field II and the others, or between fields I and III, would not be detectable using only spatial correlation or heterogeneity measures.

Using heterogeneity and spatial correlation (including SADIE) statistical measures and the arguments of Cliff and Ord (2) and Madden (26), we can summarize the type of spatial patterns found for Phomopsis leaf blight in commercial perennial plantings. About $76 \%$ of the data sets had significant overdispersion (based on $D$ ) and no significant spatial association in disease between sampling units (based on $I_{\mathrm{a}}$ ). Similar percentages were calculated based on other point-pattern statistics (e.g., $\hat{\theta})$ and correlation measures $\left(\hat{r}_{1}\right)$. This result indicates that the diseased leaflets for these data sets were aggregated (as indicated by $\rho=\theta /[1+\theta]$ ), but the typical size of the clumps was smaller than the size of the sampling units (2) (Fig. 1A to C). Considering that the disease is caused by a primarily splash-dispersed pathogen, the small scale of the diseased clumps is reasonable. Similar small-scale patterns were found for aster yellow disease of lettuce (31), based on the methods used here.

Between 12 and $18 \%$ of the data sets had significant overdispersion (heterogeneity) and spatial association, depending on whether one used first-order autocorrelation or SADIE results to determine spatial association. Here, there was a positive association in disease status of individuals within sampling units $(\rho)$ and in mean disease incidence in neighboring sampling units. These data sets could be viewed as exhibiting clumps of diseased leaflets that were larger (on average) than the size of the sampling units (2) (Fig. 1B). This type of pattern was generally associated with increased disease incidence compared with the simple overdispersion cases, which is consistent with expanding focus size as secondary spread occurs $(26,29)$, a likely result in perennial production systems. The remaining $6 \%$ of the data sets corresponded to no overdispersion and either significant positive spatial associations of disease (one data set, if $I_{\mathrm{a}}$ was used, and zero data sets, if $\hat{r}_{1}$ was used) or a regular pattern $\left(\hat{r}_{1}<0, I_{\mathrm{a}}<1\right)$. The former situation was found for Gottwald et al. (11) for plum pox virus in apricot and peach. The latter situation is representative of very small clusters of diseased leaflets, because the correlation of disease status within sampling units $(\rho)$ was 0 and sampling units with high incidence were, on average, next to sampling units with low incidence (because $\hat{r}_{1}<0$ ).

In conclusion, results showed that the incidence of Phomopsis leaf blight in the vast majority of data sets was characterized by small, loosely aggregated clusters of diseased leaflets. These clusters, on average, were typically confined within sampling units, and occasionally, the clusters extended beyond the borders of the sampling units.

\section{LITERATURE CITED}

1. Campbell, C. L., and Madden, L. V. 1990. Introduction to Plant Disease Epidemiology. John Wiley \& Sons, New York.

2. Cliff, A. D., and Ord, J. K. 1981. Spatial Processes: Models and Applications. Pion Ltd., London.

3. Collett, D. 1991. Modelling Binary Data. Chapman \& Hall, London.

4. Cressie, N. 1991. Statistics for Spatial Data. John Wiley \& Sons, New York.

5. Diggle, P. J. 1983. Statistical Analysis of Spatial Point Patterns. Academic Press, London.

6. Ellis, M. A. 1995. Integrated Pest Management (IPM). Disease Management Guidelines for Strawberries in Ohio. Ohio State Univ. Plant Pathol. Dep. Ser. 93. Ohio State University, Columbus.

7. Ellis, M. A. 1998. Strawberry Leaf Spot Diseases. Ohio State University Extension Fact Sheet HYG-3015-95. Ohio State University, Columbus.

8. Ellis, M. A., Turechek, W. W., and Madden, L. V. 1997. Evaluation of fungicides for control of strawberry leaf blight. Strawberry IPM Update 4(3):1-2. Iowa State University, Ames.

9. Ferrandino, F. J. 1998. Past nonrandomness and aggregation to spatial correlation: 2DCORR, a new approach for discrete data. Phytopathology 88:84-91.

10. Gibbons, J. D. 1985. Nonparametric Methods for Quantitative Analysis. 2nd ed. American Sciences Press, Inc., Columbus, OH.

11. Gottwald, T. R., Avinent, L., Llácer, G., Hermoso de Mendoza, A., and Cambra, M. 1995. Analysis of the spatial spread of sharka (plum pox virus) in apricot and peach orchards in eastern Spain. Plant Dis. 79:266-278.

12. Gottwald, T. R., Cambra, M., Moreno, P., Camarasa, E., and Piquer, J. 1996. Spatial and temporal analyses of citrus tristeza virus in eastern Spain. Phytopathology 86:45-55.

13. Gottwald, T. R., Reynolds, K. M., Campbell, C. L., and Timmer, L. W. 1992. Spatial and spatiotemporal autocorrelation analysis of citrus canker epidemics in citrus nurseries and groves in Argentina. Phytopathology 82:843-851.

14. Horne, J. K., and Schneider, D. C. 1995. Spatial variance in ecology. Oikos 74:18-26.

15. Hudelson, B. D., Clayton, M. K., Smith, K. P., Rouse, D. I., and Upper, C. D. 1989. Nonrandom patterns of bacterial brown spot in snap bean row segments. Phytopathology 79:674-681.

16. Hughes, G. 1988. Spatial heterogeneity in crop loss assessment models. Phytopathology 78:883-884.

17. Hughes, G., and Madden, L. V. 1992. Aggregation and incidence of disease. Plant Pathol. 41:657-660.

18. Hughes, G., and Madden, L. V. 1993. Using the beta-binomial distribution to describe aggregated patterns of disease incidence. Phytopathology 83:759-763.

19. Hughes, G., and Madden, L. V. 1994. Aggregation and incidence of disease: Some implications for sampling. Aspects Appl. Biol. 37:25-31.

20. Hughes, G., and Madden, L. V. 1995. Some methods allowing for aggregated patterns of disease incidence in the analysis of data from designed experiments. Plant Pathol. 44:927-943.

21. Hughes, G., Madden, L. V., and Munkvold, G. P. 1996. Cluster sampling for disease incidence data. Phytopathology 86:132-137.

22. Hughes, G., McRoberts, N., Madden, L. V., and Gottwald, T. R. 1997. Relationships between disease incidence at two levels in a spatial hierarchy. Phytopathology 87:542-550.

23. Hughes, G., McRoberts, N., Madden, L. V., and Nelson, S. C. 1997. Validating mathematical models of plant-disease progress in space and time. IMA (Inst. Math. Appl.) J. Math. Appl. Med. Biol. 14:85-112.

24. Hurlbert, S. H. 1984. Pseudoreplication and the design of ecological field experiments. Ecol. Monogr. 54:187-211.

25. Maas, J. L., ed. 1984. Compendium of Strawberry Diseases. The American Phytopathological Society, St. Paul, MN.

26. Madden, L. V. 1989. Dynamic nature of within-field disease and pathogen distributions. Pages 96-126 in: Spatial Components of Plant Disease Epidemics. M. J. Jeger, ed. Prentice-Hall, Englewood Cliffs, NJ.

27. Madden, L. V., and Hughes, G. 1994. BBD-Computer software for fitting the beta-binomial distribution to disease incidence data. Plant Dis. 78:536-540.

28. Madden, L. V., and Hughes, G. 1995. Plant disease incidence: Distribu- 
tions, heterogeneity, and temporal analysis. Annu. Rev. Phytopathol. 33: 529-564.

29. Madden, L. V., Hughes, G., and Ellis, M. A. 1995. Spatial heterogeneity of the incidence of grape downy mildew. Phytopathology 85:269-275.

30. Madden, L. V., Hughes, G., and Munkvold, G. P. 1996. Plant disease incidence: Inverse sampling, sequential sampling, and confidence intervals when observed mean incidence is zero. Crop Prot. 15:621-632.

31. Madden, L. V., Nault, L. R., Murral, D. J., and Apelt, M. R. 1995. Spatial pattern analysis of the incidence of aster yellows disease in lettuce. Res. Popul. Ecol. 37:279-289.

32. Madden, L. V., Pirone, T. P., and Raccah, B. 1987. Analysis of spatial patterns of virus-diseased tobacco plants. Phytopathology 77:1409-1417.

33. Madden, L. V., Reynolds, K. M., Pirone, T. P., and Raccah, B. 1988. Modeling of tobacco virus epidemics as spatio-temporal autoregressive integrated moving-average processes. Phytopathology 78:1361-1366.

34. Nault, B. A., and Kennedy, G. G. 1996. Sequential sampling plans for use in timing insecticide applications for control of European corn borer (Lepidoptera: Pyralidae) in potato. J. Econ. Entomol. 89:1468-1476.

35. Parker, S. R., Shaw, M. W., and Royle, D. J. 1997. Measurements of spatial patterns of disease in winter wheat crops and the implications for sampling. Plant Pathol. 46:470-480.

36. Perry, J. N. 1995. Spatial aspects of animal and plant distribution in patchy farmland habitats. Pages 221-242 in: Ecology and Integrated Farming Systems. D. M. Glen, M. P. Greaves, and H. M. Anderson, eds. John Wiley \& Sons, London.

37. Perry, J. N. 1995. Spatial analysis by distance indices. J. Anim. Ecol. 64: 303-314.

38. Perry, J. N. 1998. Measures of spatial pattern for counts. Ecology 79: 1008-1017.

39. Perry, J. N., and Hewitt, M. 1991. A new index of aggregation for animal counts. Biometrics 47:1505-1518.

40. Perry, J. N., and Taylor, L. R. 1985. Adès: New ecological families of species-specific frequency distributions that describe repeated spatial samples with an intrinsic power-law variance-mean property. J. Anim. Ecol. 54:931-953.

41. Pielou, E. C. 1977. Mathematical Ecology. John Wiley \& Sons, New York.

42. Reed, W. J. 1983. Confidence estimation of ecological aggregation indices based on counts-A robust procedure. Biometrics 39:987-998.

43. Reynolds, K. M., and Madden, L. V. 1988. Analysis of epidemics using spatio-temporal autocorrelation. Phytopathology 78:240-246.

44. Reynolds, K. M., Madden, L. V., and Ellis, M. A. 1988. Spatio-temporal analysis of epidemic development of leather rot of strawberry. Phytopathology 78:246-252.

45. Schotzko, D. J., and Knudsen, G. R. 1992. Use of geostatistics to evaluate a spatial simulation of Russian wheat aphid (Homoptera: Aphididae) movement behavior on preferred and nonpreferred hosts. Environ. Entomol. 21:1271-1282.

46. Sun, P., and Madden, L. V. 1997. Using a normal approximation to test for the binomial distribution. Biom. J. 39:533-544.

47. Tanne, E., Marcus, R., Dubitzky, E., and Raccah, B. 1996. Analysis of progress and spatial pattern of corky bark in grape. Plant Dis. 80:34-38.

48. Tarone, R. E. 1979. Testing the goodness of fit of the binomial distribution. Biometrika 66:585-590.

49. Taylor, L. R. 1961. Aggregation, variance and the mean. Nature (Lond.) 189:732-735.

50. Upton, G., and Fingleton, B. 1985. Spatial Data Analysis by Example, Vol. 1. Point Pattern and Quantitative Data. John Wiley \& Sons, Chichester, West Sussex, England.

51. Williams, D. A. 1975. The analysis of binary responses from toxicological experiments involving reproduction and teratogenicity. Biometrics 31:949-952.

52. Xiao, C. L., Hao, J. J., and Subbarao, K. V. 1997. Spatial patterns of microsclerotia of Verticillium dahliae in soil and verticillium wilt of cauliflower. Phytopathology 87:325-331. 